\title{
In-Flight Multichannel Calibration for Along-Track Interferometric Airborne Radar
}

\author{
André Barros Cardoso da Silva ${ }^{\circledR}$, Stefan Valentin Baumgartner ${ }^{\circledR}$, Member, IEEE, \\ Felipe Queiroz de Almeida ${ }^{(}$, and Gerhard Krieger ${ }^{\circledR}$, Fellow, IEEE
}

\begin{abstract}
Multichannel calibration is essential for detecting moving targets and for estimating their positions and velocities accurately. This article presents a fast and efficient calibration algorithm for the along-track multichannel systems, in particular for space-time adaptive processing (STAP) techniques. The proposed algorithm corrects the phase and magnitude offsets of the receive channels and also takes into account the Doppler centroid variation (e.g., caused by atmospheric turbulences) along the slant range and the azimuth time. The knowledge of the Doppler centroid variation is especially important for an accurate clutter covariance matrix estimation, which is required by STAP for efficient clutter suppression. Important calibration parameters and offsets are estimated directly from the range-compressed training data. The proposed algorithm is evaluated based on real multichannel X-band radar data acquired with DLR's airborne system F-SAR and compared with the state-of-the-art digital channel balancing technique. The experimental results show the potential of the proposed calibration algorithm toward real-time applications.
\end{abstract}

Index Terms-Airborne radar, calibration, ground moving target indication (GMTI), radar applications, radar signal processing, synthetic aperture radar (SAR), traffic monitoring.

\section{INTRODUCTION}

$\mathbf{I}$ $\mathrm{N}$ PRACTICE, it is not possible to build absolutely identical antennas and receive channels with the same electrical characteristics and time delays. Thus, the transfer functions and the antenna gain patterns of the receive channels differ from each other and need to be characterized or equalized [1], especially for multichannel techniques that make use of the sum and difference channels.

For applications on ground moving target indication (GMTI), the different transmitting (TX) and receiving (RX) antenna characteristics can be measured or estimated and then incorporated directly into the direction-of-arrival (DOA) vector. Normally, external calibration can be used for compensating different time delays between multiple RX channels (e.g., using reference targets or special calibration loops in the radar hardware design). Moreover, the precise knowledge of the along-track baselines between multiple RX channels is

Manuscript received April 20, 2020; revised July 8, 2020; accepted July 13, 2020. (Corresponding author: André Barros Cardoso da Silva.)

The authors are with the Radar Concepts Department, Microwaves and Radar Institute, German Aerospace Center (DLR), 82234 Oberpfaffenhofen, Germany (e-mail: andre.silva@dlr.de; stefan.baumgartner@dlr.de; felipe.queirozdealmeida@dlr.de; gerhard.krieger@dlr.de).

Color versions of one or more of the figures in this article are available online at http://ieeexplore.ieee.org.

Digital Object Identifier 10.1109/TGRS.2020.3011287 important for estimating the DOA angles accurately, which affect the position and the velocity estimates of the targets.

An elegant and robust method for digital channel balancing (DCB) was introduced in [2] and discussed in detail in [1]. In this method, the RX channels are balanced with respect to a reference RX channel by performing an iterative approach in the 2-D frequency domain, so that the residual phase and magnitude offsets are compensated. Furthermore, the alongtrack baselines among the RX channels can be estimated accurately in the range-Doppler domain [1].

An interesting review about the calibration techniques was presented in [3], in which simple algorithms (e.g., based on 1-D and 2-D co-registrations) and more sophisticated methodologies (e.g., the DCB) were evaluated in detail for alongtrack interferometry (ATI) [4], displaced phase center antenna (DPCA) [5], and space-time adaptive processing (STAP) [6] techniques. It is shown in [3] that the DCB achieves the best performance mainly in terms of clutter suppression at the expense of a much higher computational effort compared with other methodologies.

More recently, an external calibration algorithm for multichannel airborne synthetic aperture radar (SAR) systems was presented in [7], through which it is possible to estimate accurately the antenna baselines and attitude angles, among other parameters. The algorithm is applied on the rangecompressed data (no azimuth compression is needed) and relies on a previously measured antenna model for proper operation. It was designed for the new DLR's airborne digitalbeamforming system DBF-SAR [8].

This article presents a fast and efficient calibration algorithm for multichannel airborne SAR systems that is able to 1) correct the phase and magnitude offsets among multiple RX channels with respect to a reference channel and 2) estimate and compensate the Doppler centroid variation (e.g., caused by atmospheric turbulences) along the slant range and the azimuth time using the attitude angles of the antenna array. The Doppler centroid compensation is especially important in the scope of this article because the post-Doppler (PD) STAP technique (see the Appendix) is used for GMTI. The PD STAP requires Doppler centroid compensation to obtain an accurate clutter covariance matrix (CCM) estimation, whose accuracy impacts the clutter suppression capability. Important parameters and offsets are estimated directly from the range-compressed training data, and for the GMTI application considered in this article, the estimation of such parameters 
and offsets does not require an antenna model (i.e., assuming that absolute radiometric accuracy is not required). Moreover, once estimated, the parameters and the offsets can be stored in the memory and be directly applied for the calibration of subsequent flights with acceptable performance, thus speeding up the processing time.

The proposed calibration algorithm is compared with the state-of-the-art DCB technique [1], [2] in terms of processing time, phase correction accuracy, and GMTI results (see Section VI). The established DCB technique is principally chosen for comparison since it is well-known in the literature and is still used for calibrating the radar data acquired with the state-of-the-art multichannel GMTI systems [9]. The main benefits of the proposed calibration algorithm are presented in Section III-A.

The remainder of this article is organized as follows. Section II presents the signal model for the PD STAP technique and examples of the expected errors that impact the target position estimation accuracy. Section III presents the benefits and the main processing steps of the proposed calibration algorithm. Section IV presents the mathematical framework necessary for estimating important parameters and offsets that are required by the proposed calibration algorithm. Section V introduces the mathematical framework needed for correcting channel imbalances using the previously estimated offsets and parameters. Section VI evaluates the proposed calibration algorithm based on real multichannel X-band radar data sets acquired with DLR's airborne system F-SAR [10], [11], comparing it with the state-of-the-art DCB technique. This article is concluded with a short summary and discussion in Section VII.

\section{Signal Model And Expected ERrors}

In this article, the PD STAP technique (see the main features in the Appendix) is considered for evaluating the proposed calibration algorithm in terms of GMTI performance (see Section VI-D). The remainder of Section II presents the signal model for the PD STAP technique and introduces important terms. Finally, examples of the expected errors are presented and simulated, highlighting the need for calibration.

\section{A. Signal Model}

The multichannel signal model for the PD STAP technique can be expressed by [12]

$$
\begin{aligned}
\mathbf{s}(t)= & a_{\mathrm{s}} e^{-j \frac{4 \pi}{\lambda} R(t)} D_{\mathrm{tx}}\left[u_{\text {array }}(t)\right] \\
& \times\left[\begin{array}{c}
D_{\mathrm{rx}, 1}\left[u_{\text {array }}(t)\right] e^{j \frac{2 \pi}{\lambda} u_{\text {array }}(t) x_{1}} \\
D_{\mathrm{rx}, 2}\left[u_{\text {array }}(t)\right] e^{j \frac{2 \pi}{\lambda} u_{\text {array }}(t) x_{2}} \\
\vdots \\
D_{\mathrm{rx}, M}\left[u_{\text {array }}(t)\right] e^{j \frac{2 \pi}{\lambda} u_{\text {array }}(t) x_{\mathrm{M}}}
\end{array}\right] \\
= & a_{\mathrm{s}} e^{-j \frac{4 \pi}{\lambda} R(t)} d\left[u_{\text {array }}(t)\right] \in \mathbb{C}^{M \times 1}
\end{aligned}
$$

where $a_{\mathrm{s}}$ is a complex value that accounts for the reflectivity of the scatterer, $\lambda$ is the radar wavelength, $M$ is the number of RX channels, $R(t)$ is the range to the antenna array

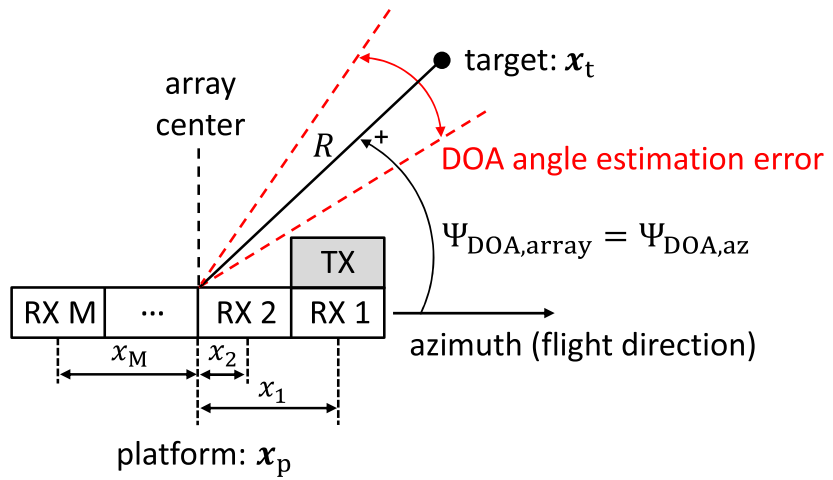

Fig. 1. Acquisition geometry considering a multichannel antenna and zero squint angle. In practice, channel imbalances may cause an error on the target DOA angle estimation.

center, $D_{\mathrm{tx}}\left[u_{\text {array }}(t)\right]$ and $D_{\mathrm{rx}, \mathrm{m}}\left[u_{\text {array }}(t)\right]$ are the complex TX and RX azimuth antenna characteristics of the $m$ th channel, respectively, $x_{\mathrm{m}}$ is the position of the antenna center in the azimuth direction with respect to the antenna array center (see Fig. 1$), \boldsymbol{d}\left[u_{\text {array }}(t)\right]$ is the beamforming or DOA vector, $u_{\text {array }}=\cos \left(\Psi_{\mathrm{DOA} \text {,array }}\right)$ is the directional cosine, and $\Psi_{\text {DOA, array }}$ is the DOA angle of the target measured with respect to the antenna array axis.

Fig. 1 shows the acquisition geometry of a multichannel antenna, for which the antenna array center origin is arbitrarily chosen at the center of the array, so that $\sum_{\mathrm{m}} x_{\mathrm{m}}=0$. The DOA angle of the target measured with respect to the azimuth or flight direction is denoted by $\Psi_{\mathrm{DOA}, \mathrm{az}}$. Note that the antenna array and the azimuth axes coincide in the shown example since the squint angle is considered zero (for simplicity), so that $\Psi_{\mathrm{DOA} \text {,array }}=\Psi_{\mathrm{DOA}, \text { az }}$. The ground coordinates of the target are $\boldsymbol{x}_{\mathrm{t}}=\left[x_{\mathrm{t}}, y_{\mathrm{t}}, z_{\mathrm{t}}\right]^{T}$, where the symbol $[\cdot]^{T}$ denotes the transpose operator. The coordinates of the platform are given by $\boldsymbol{x}_{\mathrm{p}}=\left[x_{\mathrm{p}}, y_{\mathrm{p}}, z_{\mathrm{p}}\right]^{T}$.

The coordinates of the target in azimuth $x_{\mathrm{t}}$ and in ground range $y_{\mathrm{t}}$ can be expressed, respectively, as [9]

$$
\begin{aligned}
& x_{\mathrm{t}}=x_{\mathrm{p}}+R \cdot \cos \left(\Psi_{\mathrm{DOA}, \mathrm{az}}\right) \\
& y_{\mathrm{t}}=y_{\mathrm{p}}+\sqrt{\left(R \cdot \sin \left(\Psi_{\mathrm{DOA}, \mathrm{az}}\right)\right)^{2}-\left(z_{\mathrm{p}}-z_{\mathrm{t}}\right)^{2}}
\end{aligned}
$$

where the altitude of the target $z_{\mathrm{t}}$ can be obtained for instance from a digital elevation model, which is especially important in case of high topographic slopes [13].

Fig. 1 shows (in red) a potential error on the target DOA angle estimation that in practice may be caused by the phase offsets, which need to be estimated and corrected by a proper calibration algorithm (e.g., the algorithm presented in Section III). Note that the target DOA angle impacts principally the estimation of the target position, as it can be seen from (2) and (3).

\section{B. Errors Due to Incorrect Yaw Angle}

A major challenge for most airborne SAR systems is to cope with atmospheric turbulences, which induce errors on the aircraft motion angles: yaw, pitch, and roll (described in detail in Section IV-D, see Fig. 11). In particular, the yaw and the 
pitch angles have a prominent impact on the Doppler centroid, which plays an important role for GMTI applications.

The Doppler centroid and the yaw angle can be related as

$$
f_{\mathrm{DC}, \mathrm{YAW}}=\frac{2 \cdot v_{\mathrm{p}}}{\lambda}\left[\sin \left(\theta_{\mathrm{i}}\right) \cdot \tan \left(\theta_{\mathrm{YAW}, \mathrm{IMU}}+\Delta \theta_{\mathrm{YAW}}\right)\right]
$$

where $v_{\mathrm{p}}$ denotes the velocity of the platform, $\theta_{\mathrm{YAW}, \mathrm{IMU}}$ denotes the yaw angle obtained from the aircraft's inertial measurement unit (IMU), and $\theta_{\mathrm{i}}$ denotes the incidence angle (the flat earth assumption is used). The term $\Delta \theta_{\mathrm{YAW}}$ denotes an offset that in practice may exist due to a skewed antenna mount on the fuselage and due to the distance between the IMU and the antenna, known as "lever arms" (see Fig. 11).

A misestimate of the yaw angle offset impacts the Doppler centroid. This impact can be verified by error propagation, from which the standard deviation of the Doppler centroid can be obtained according to

$$
\begin{aligned}
& \sigma_{f_{\mathrm{DC}, \mathrm{YAW}}} \\
& =\sqrt{\left(\frac{\partial f_{\mathrm{DC}, \mathrm{YAW}}}{\partial \Delta \theta_{\mathrm{YAW}}}\right)^{2} \cdot \sigma_{\Delta \theta_{\mathrm{YAW}}}^{2}} \\
& =\sqrt{\left(\frac{2 \cdot v_{\mathrm{p}}}{\lambda}\left[\sin \left(\theta_{\mathrm{i}}\right) \cdot \sec ^{2}\left(\theta_{\mathrm{YAW}, \mathrm{IMU}}+\Delta \theta_{\mathrm{YAW}}\right)\right]\right)^{2} \cdot \sigma_{\Delta \theta_{\mathrm{YAW}}^{2}}}
\end{aligned}
$$

where $\sigma_{\Delta \theta_{\mathrm{YAW}}}$ denotes the standard deviation of the yaw angle offset estimation.

Fig. 2(a) shows the expected Doppler centroid error $\sigma_{f_{\mathrm{DC}, \mathrm{YAW}}}$, which was obtained with (5) for different incidence angles and for different estimation errors of the yaw angle offset. In this example, the following simulation parameters were assumed: $\lambda=0.03125 \mathrm{~m}, v_{\mathrm{p}}=90 \mathrm{~m} / \mathrm{s}, \theta_{\mathrm{YAW}, \mathrm{IMU}}=0^{\circ}, \Delta \theta_{\mathrm{YAW}}=0^{\circ}$, $\theta_{\mathrm{i}}=\left[25^{\circ}, 63^{\circ}\right]$, and $\sigma_{\Delta \theta_{\mathrm{YAW}}}=\left[0^{\circ}, 1^{\circ}\right]$. As can be noticed in Fig. 2(a), a Doppler centroid error of about $\sigma_{f_{\mathrm{DC}, \text { YAW }}}=90 \mathrm{~Hz}$ can be expected in the far range $\left(\theta_{\mathrm{i}}=63^{\circ}\right)$ for a yaw angle offset accuracy of $\sigma_{\Delta \theta_{\mathrm{YAW}}}=1^{\circ}$.

The Doppler centroid can generally be related to the directional cosine $u_{\text {array }}=\cos \left(\Psi_{\mathrm{DOA} \text {,array }}\right)$ and the line-of-sight velocity $v_{\mathrm{r}}$ of the target according to [14]

$$
u_{\text {array }}=\frac{\lambda}{2 \cdot v_{\mathrm{p}}} \cdot\left(f_{\mathrm{a}}-f_{\mathrm{DC}}\right)+\frac{v_{\mathrm{r}}}{v_{\mathrm{p}}}
$$

where in particular $f_{\mathrm{DC}}=f_{\mathrm{DC}, \mathrm{YAW}}$ can be assumed.

Note from (6) that a Doppler centroid error impacts the directional cosine (or the DOA angle) estimation of the target. This impact can be verified by means of error propagation: the standard deviation of the directional cosine can be generally obtained according to

$$
\sigma_{u_{\text {array }}}=\sqrt{\left(\frac{\partial u_{\text {array }}}{\partial f_{\mathrm{DC}}}\right)^{2} \cdot \sigma_{f_{\mathrm{DC}}}^{2}}=\sqrt{\left(\frac{\lambda}{2 \cdot v_{\mathrm{p}}}\right)^{2} \cdot \sigma_{f_{\mathrm{DC}}}^{2}}
$$

where in particular $\sigma_{f_{\mathrm{DC}}}=\sigma_{f_{\mathrm{DC}, \text { YAW }}}$ is obtained from (5).

Finally, an error on the directional cosine (or DOA angle) impacts the target's azimuth position, as it can be seen in (2). This impact can be verified once more by means of error propagation: the standard deviation of the target azimuth
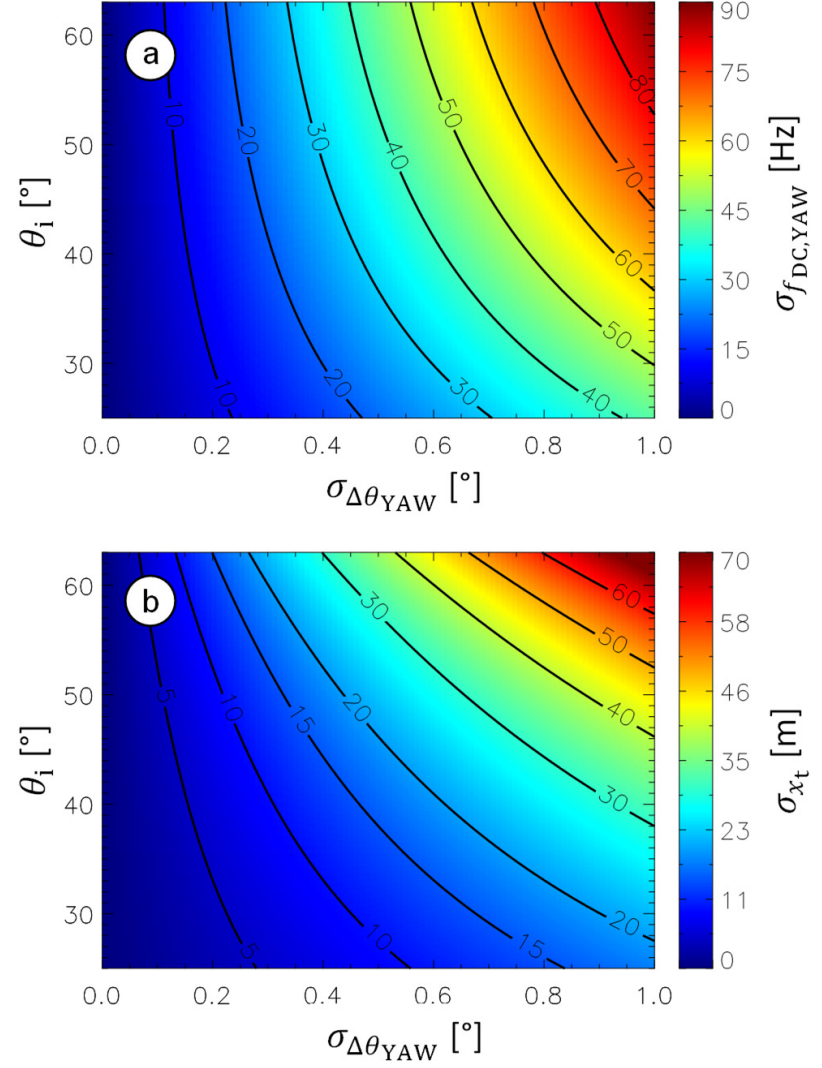

Fig. 2. Errors expected for (a) Doppler centroid $\sigma_{f \mathrm{DC}, \text { YAW }}[\mathrm{Hz}]$ and (b) target azimuth position $\sigma_{x_{\mathrm{t}}}[\mathrm{m}]$. The errors were estimated as a function of the incidence angles $\theta_{\mathrm{i}}$ and for different estimation errors of the yaw angle offset $\sigma_{\Delta \theta_{\mathrm{YAW}}}$.

position can be generally obtained according to

$$
\sigma_{x_{\mathrm{t}}}=\sqrt{\left(\frac{\partial x_{\mathrm{t}}}{\partial u_{\text {array }}}\right)^{2} \cdot \sigma_{u_{\text {aray }}^{2}}^{2}}=\sqrt{(R)^{2} \cdot \sigma_{u_{\text {aray }}^{2}}^{2}} .
$$

Fig. 2(b) shows the expected target azimuth error $\sigma_{x_{\mathrm{t}}}$, which was obtained with (8) for different incidence angles and for different estimation errors of the yaw angle offset. In this example, the same parameters as considered for Fig. 2(a) were used and $R=h / \cos \left(\theta_{\mathrm{i}}\right)$, where $h=2200 \mathrm{~m}$ was set as the flight altitude. From Fig. 2(b), it can be seen that a target azimuth position error of about $\sigma_{x_{\mathrm{t}}}=70 \mathrm{~m}$ can be expected in far range $\left(\theta_{\mathrm{i}}=63^{\circ}\right)$ for a yaw angle offset accuracy of $\sigma_{\Delta \theta_{\mathrm{YAW}}}=1^{\circ}$.

The example shown in Fig. 2 takes into account only the estimation error of the yaw angle offset, which results in a Doppler centroid error [see Fig. 2(a)] that can cause a significant impact on the target azimuth position estimation [see Fig. 2(b)].

\section{Errors Due to Incorrect Pitch Angle}

The Doppler centroid and the pitch angle can be related as

$$
f_{\mathrm{DC}, \mathrm{PITCH}}=\frac{2 \cdot v_{\mathrm{p}}}{\lambda}\left[\cos \left(\theta_{\mathrm{i}}\right) \cdot \tan \left(\theta_{\mathrm{PITCH}, \mathrm{IMU}}+\Delta \theta_{\mathrm{PITCH}}\right)\right]
$$

where $\theta_{\mathrm{PITCH}, \mathrm{IMU}}$ denotes the pitch angle obtained from the IMU and $\Delta \theta_{\text {PITCH }}$ denotes a pitch offset. 

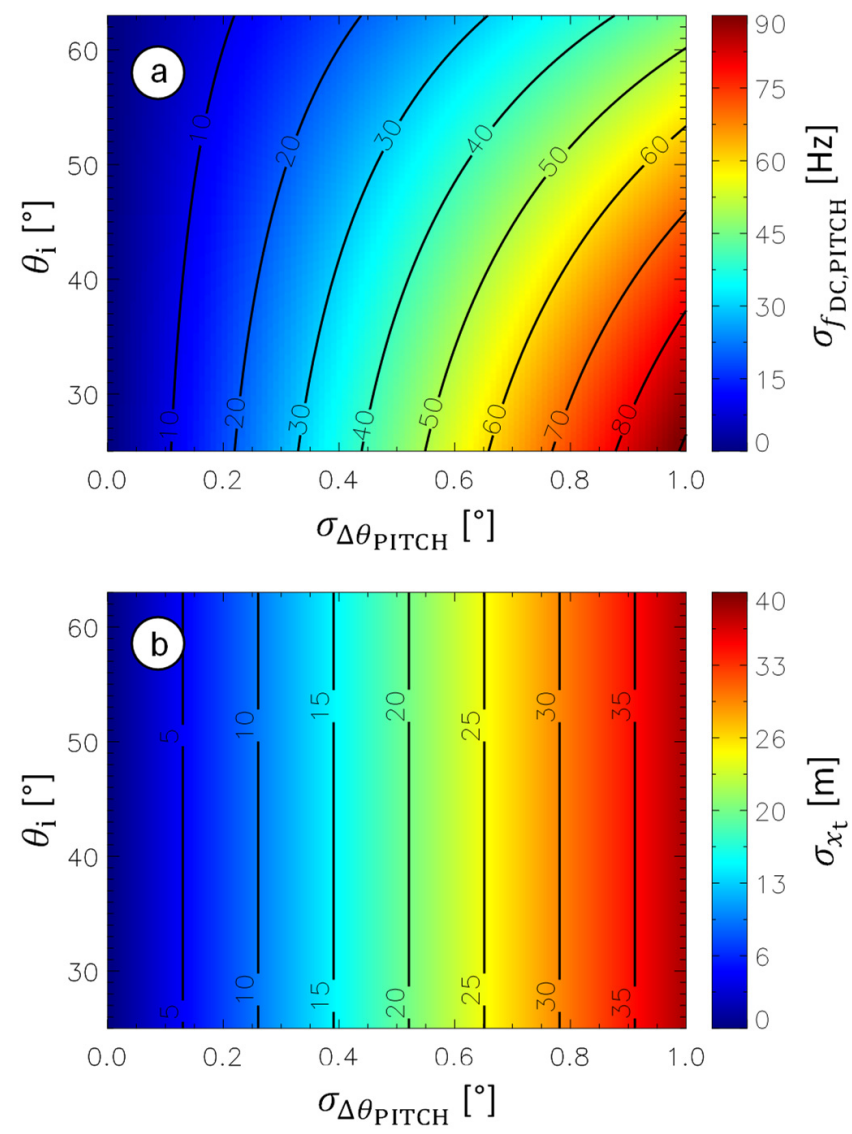

Fig. 3. Errors expected for (a) Doppler centroid $\sigma_{f_{\mathrm{DC} \text {. PTCH }}}[\mathrm{Hz}]$ and (b) target azimuth position $\sigma_{x_{\mathrm{t}}}[\mathrm{m}]$. The errors were estimated as a function of the incidence angles $\theta_{\mathrm{i}}$ and for different estimation errors of the pitch angle offset $\sigma_{\Delta \theta_{\mathrm{PITCH}}}$.

As for the yaw angle in Section II-B, a misestimate of the pitch angle offset also impacts the Doppler centroid. This impact can be verified by error propagation: the standard deviation of the Doppler centroid can be obtained according to

$$
\begin{aligned}
& \sigma_{f_{\mathrm{DC}, \mathrm{PITCH}}} \\
& =\sqrt{\left(\frac{\partial f_{\mathrm{DC}, \mathrm{PITCH}}}{\partial \Delta \theta_{\mathrm{PITCH}}}\right)^{2} \cdot \sigma_{\Delta \theta_{\mathrm{PITCH}}}^{2}} \\
& =\sqrt{\left(\frac{2 \cdot v_{\mathrm{p}}}{\lambda}\left[\cos \left(\theta_{\mathrm{i}}\right) \cdot \sec ^{2}\left(\theta_{\mathrm{PITCH}, \mathrm{IMU}}+\Delta \theta_{\mathrm{PITCH}}\right)\right)^{2} \cdot \sigma_{\Delta \theta_{\mathrm{PITCH}}}^{2}\right.}
\end{aligned}
$$

where $\sigma_{\Delta \theta_{\mathrm{PITH}}}$ denotes the standard deviation of the pitch angle offset estimation.

Fig. 3(a) shows the expected Doppler centroid error $\sigma_{f_{\mathrm{DC}} \text { PITCH }}$, which was obtained according to (10) for different incidence angles and for different estimation errors of the pitch angle offset. The same parameters assumed for Section II-B were considered in this example together with $\theta_{\mathrm{PITCH}, \mathrm{IMU}}=0^{\circ}, \Delta \theta_{\mathrm{PITCH}}=0^{\circ}$ and $\sigma_{\Delta \theta_{\mathrm{PITCH}}}=\left[0^{\circ}, 1^{\circ}\right]$. As can be seen in Fig. 3(a), a Doppler centroid error of about $\sigma_{f_{\mathrm{DC} \text {, PTTCH }}}=90 \mathrm{~Hz}$ can be expected in the near range $\left(\theta_{\mathrm{i}}=25^{\circ}\right)$ for a pitch angle offset accuracy of $\sigma_{\Delta \theta_{\mathrm{PITCH}}}=1^{\circ}$.

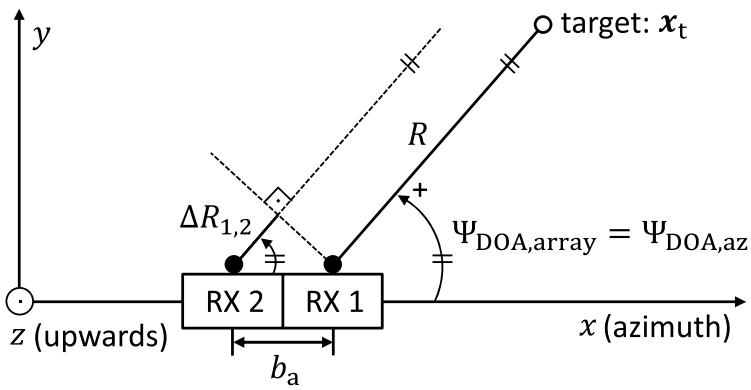

Fig. 4. Acquisition geometry considering an array with two RX channels. The squint angle is assumed zero for simplicity.

The impact of the Doppler centroid error $\sigma_{f_{\mathrm{DC} \text {,PTCH }} \text { on }}$ the directional cosine can be obtained from (7) by setting $\sigma_{f_{\mathrm{DC}}}=\sigma_{f_{\mathrm{DC}, \mathrm{PTTC}}}$. Then, the impact of the directional cosine (or DOA angle) on the target azimuth position can be obtained from (8).

Fig. 3(b) shows the expected target azimuth error $\sigma_{x_{\mathrm{t}}}$, which was obtained with (8) for different incidence angles and for different estimation errors of the pitch angle offset. The same parameters considered for Section II-B were used in this example. From Fig. 3(b), it can be seen that a target azimuth position error of about $\sigma_{x_{\mathrm{t}}}=40 \mathrm{~m}$ can be expected for a pitch angle offset accuracy of $\sigma_{\Delta \theta_{\text {PाTC }}}=1^{\circ}$, which is independent of the incidence angles.

\section{Errors Due to Incorrect Roll Angle}

Generally, a roll angle around the antenna array center axis causes a negligible impact on the Doppler centroid and therefore on the target azimuth position.

Nevertheless, since the antenna array is mounted on the aircraft's fuselage, an offset exists (known as "lever arms," see Fig. 11) between the aircraft and the IMU coordinates' origin. Due to this offset, an aircraft roll may also introduce additional pitch and yaw angle components in the reference system of the antenna array. This could be avoided if the array is mounted perfectly aligned with the aircraft longitudinal axis, which is not feasible in practice.

Anyhow, the roll angle contributions to the antenna array pitch and yaw offset angles are covered by the already discussed $\Delta \theta_{\text {YAW }}$ and $\Delta \theta_{\text {PITCH }}$ terms. These terms are estimated and corrected by the proposed calibration method presented in Section III.

\section{E. Errors Due to Inaccurate Baseline Estimation}

In practice, the along-track distances between the RX channels (also denominated along-track baselines) need to be estimated accurately from the measured data, as presented in detail in Section IV-C.

Consider the acquisition geometry as shown in Fig. 4, in which the antenna array has two RX channels (RX1 and $\mathrm{RX} 2), b_{\mathrm{a}}$ denotes the physical along-track baseline and $\Delta R_{1,2}$ denotes the difference between the ranges of the target to each of the RX channel. The squint angle is assumed zero for simplicity. The antenna array is perfectly aligned without 


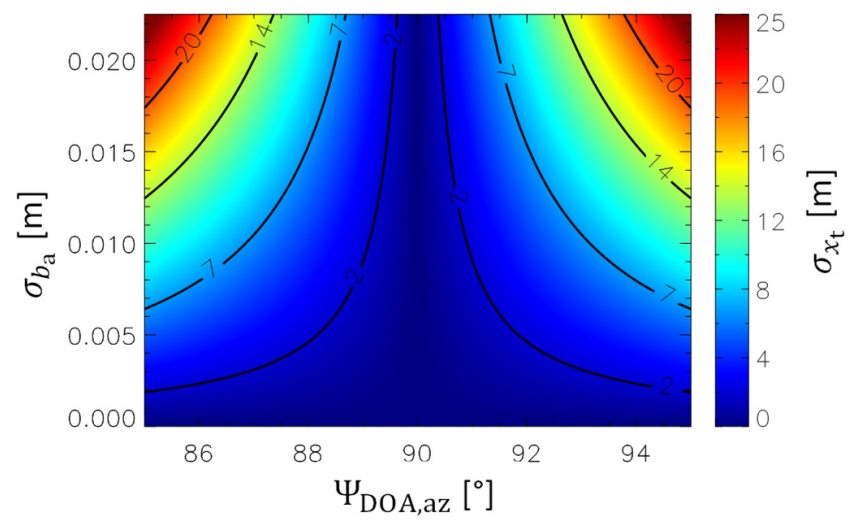

Fig. 5. Expected target azimuth position error caused by baseline estimation errors and for different target DOA angles.

any influence of the attitude angles, so that the DOA angle $\Psi_{\mathrm{DOA} \text {,array }}=\Psi_{\mathrm{DOA}, \mathrm{az}}$. A stationary target $\boldsymbol{x}_{t}$ is located in far field at a slant range $R$.

In the example shown in Fig. 4 , the range difference $\Delta R_{1,2}$ is related to the directional cosine $u_{\text {array }}$ according to

$$
u_{\text {array }}=\cos \left(\Psi_{\mathrm{DOA}, \text { array }}\right)=\frac{\Delta R_{1,2}}{b_{\mathrm{a}}} .
$$

The estimation error of the along-track baseline impacts the directional cosine. This impact can be verified once more by error propagation: the standard deviation of the directional cosine can be obtained by

$$
\sigma_{u_{\text {aray }}}=\sqrt{\left(\frac{\partial u_{\text {array }}}{\partial b_{\mathrm{a}}}\right)^{2} \cdot \sigma_{b_{\mathrm{a}}}^{2}}=\sqrt{\left(\frac{\Delta R_{1,2}}{\left(b_{\mathrm{a}}\right)^{2}}\right)^{2} \cdot \sigma_{b_{\mathrm{a}}}^{2}}
$$

where $\sigma_{b_{\mathrm{a}}}$ denotes the standard deviation of the along-track baseline estimation.

Finally, the impact of the directional cosine (or DOA angle) on the target azimuth position can be obtained by substituting (12) into (8).

Fig. 5 shows the expected target azimuth position error $\sigma_{x_{\mathrm{t}}}$ obtained for different baseline estimation errors $\sigma_{b_{\mathrm{a}}}$ and for different DOA angles $\Psi_{\mathrm{DOA}, \mathrm{az}}$. In this example, the acquisition geometry shown in Fig. 4 was considered, in which the physical baseline was $b_{\mathrm{a}}=0.2 \mathrm{~m}$, the flight altitude was $h=2200 \mathrm{~m}$, and the platform velocity was $v_{\mathrm{p}}=90 \mathrm{~m} / \mathrm{s}$. The stationary target was located at a fixed slant range $R=2427 \mathrm{~m}$ and at a varying DOA angle $\Psi_{\mathrm{DOA}, \mathrm{az}}=\left[85^{\circ}, 95^{\circ}\right]$.

For instance, it can be seen in Fig. 5 that a baseline estimation error of $\sigma_{b_{\mathrm{a}}}=0.025 \mathrm{~m}$ can cause a target azimuth error in the order of $\sigma_{x_{\mathrm{t}}}=25 \mathrm{~m}$ for a DOA angle of $\Psi_{\text {DOA,az }}=85^{\circ}$ (i.e., in the top-left corner of Fig. 5). It can also be seen by the contour lines that the expected target azimuth error is better than $2 \mathrm{~m}$ in case $\sigma_{b_{a}}=0.002 \mathrm{~m}$ (i.e., if the baselines are accurately estimated).

\section{F. First-Order Motion Compensation}

For the results presented in this article, it is important to mention that a first-order motion compensation algorithm was

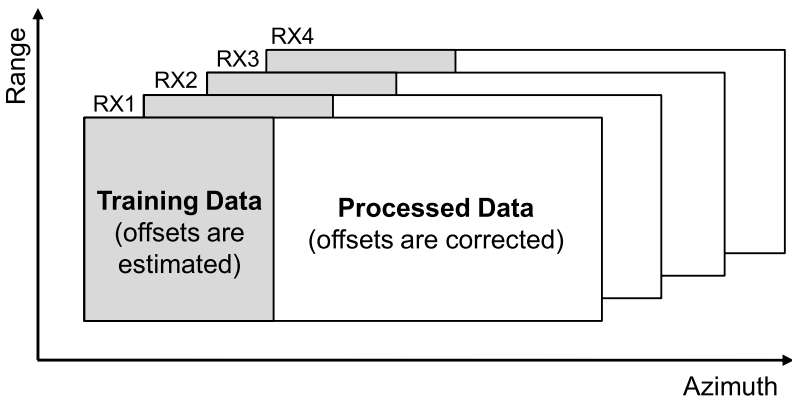

Fig. 6. Example of multichannel radar data. The training data are collected at the beginning of the flight for parameter estimation. Then, the parameters are stored in the memory and directly applied for calibrating the next acquired radar data.

applied in a preprocessing step [15]. This algorithm is carried out directly with the range-uncompressed data with the aim of correcting phase errors with respect to a reference range. After the first-order motion compensation, the platform trajectory is corrected to a straight line only for the reference range. The velocity variations in the aircraft are also compensated so that constant pixel spacing in azimuth can be achieved.

\section{Proposed Calibration Algorithm}

Calibration is essential for obtaining accurate position and velocity estimates of the moving targets. Section III introduces the benefits of the proposed calibration algorithm together with an overview of the main processing blocks. In addition, a calibration algorithm based on the state-of-the-art DCB is presented, which is used as reference in this article for comparison with the proposed calibration algorithm.

\section{A. Main Benefits}

The proposed algorithm requires the estimation of important parameters and offsets to obtain accurate DOA angle estimation of the moving targets.

The main idea is depicted in Fig. 6. The estimation of parameters and offsets is carried out at the beginning of the flight using homogeneous multichannel training data. Then, the estimated parameters and offsets are stored in the memory and are directly applied for calibrating the next radar data acquired during the same flight, thus speeding up the processing time.

The parameters and offsets can even be estimated once in a flight campaign (e.g., during the calibration flight) and be applied on subsequent flights if the antenna pod is not re-assembled on the aircraft's fuselage. This is an important benefit of the proposed algorithm, which makes it especially suitable for applications that require in-flight calibration.

The proposed algorithm also has great potential for maritime applications, although not discussed in the scope of this article. In this case, the parameters and offsets can be estimated once using land clutter (homogeneous multichannel training data), stored in the memory, and then applied for calibrating the radar data acquired over the ocean. 


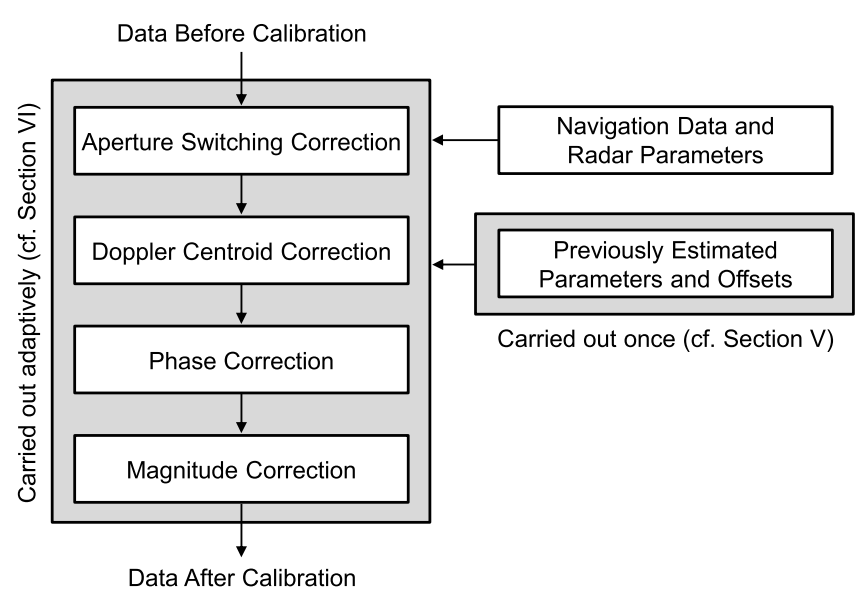

Fig. 7. Main steps of the proposed calibration algorithm. The channel imbalance correction (gray box on the left) is carried out adaptively using the previously estimated parameters and offsets (gray box on the right).

\section{B. Main Processing Blocks}

The simplified processing chain of the proposed calibration algorithm is depicted in Fig. 7. The gray box on the left shows the correction of the channel imbalances, which is carried out adaptively by considering four main processing blocks: aperture switching (AS) correction, Doppler centroid correction, phase correction, and magnitude correction. The framework necessary for the channel imbalance correction is presented in Section V.

The channel imbalance correction requires the navigation data of the aircraft (e.g., position, velocity, attitude angles, and heading angle), the radar parameters, and the important parameters and offsets (see the gray box on the right in Fig. 7) that need to be estimated at the beginning of the flight or in a previous flight. The framework necessary for the parameter and offset estimation is presented in detail in Section IV.

In Fig. 7, the first correction step consists in compensating a potential time delay that is introduced by the antenna AS technique, which is used by the DLR's airborne system F-SAR for creating additional phase centers (see Section V-A). Naturally, this operation is omitted in case of systems that do not use the AS technique.

In the Doppler centroid correction block, all range bins of the measured data are shifted to Doppler zero by taking into account the range-dependent Doppler centroid (known in the literature as "J-Hook" [16]) and the Doppler centroid variation along the azimuth time caused by the motion of the aircraft (see Fig. 11). The Doppler centroid correction (see Section V-B) is especially important for the PD STAP technique to remove the J-Hook that directly affects the CCM estimation (see the Appendix). Indeed, note that in (40) the term $\hat{\boldsymbol{R}}_{\mathrm{W}}$ is estimated for each Doppler frequency bin $f_{\mathrm{a}}$ by performing an averaging along the slant range direction. Other GMTI techniques may not require Doppler centroid correction, and in such cases this operation can be omitted.

In the phase correction block, the phases are corrected by taking into account the squint angle variation as a function of the incidence and attitude angles: yaw, pitch, and roll. After the phase correction (see Section V-C), the phase distributions are

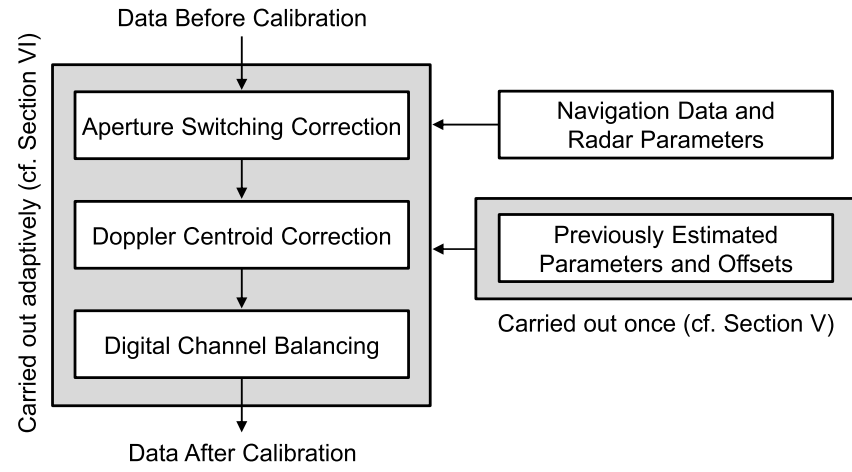

Fig. 8. Main steps of the calibration algorithm based on the state-ofthe-art DCB technique, which is compared with the proposed algorithm in Section VI. The channel imbalance correction (gray box on the left) is carried out adaptively using previously estimated parameters and offsets (gray box on the right).

centered around zero degrees, as it can be seen in Fig. 18(e). Such a distribution indicates a successful correction.

In the Magnitude Correction block, the differences in the RX channel magnitudes are compensated by applying the previously estimated magnitude offsets (see Sections IV-B and V-D).

\section{Version Based on DCB}

The proposed calibration algorithm can be modified to use the state-of-the-art DCB technique. [1], [2] for correcting the phase and magnitude offsets. In this case, such offsets are corrected through a computationally expensive operation that is carried out adaptively in the 2-D Doppler frequency domain. This approach is taken in this article as a reference to evaluate the proposed calibration algorithm in terms of processing time, phase correction accuracy, and GMTI results (see Section VI).

The reference processing chain is shown in Fig. 8, which is similar to the one shown in Fig. 7, except that the blocks phase correction and magnitude correction in Fig. 7 are exchanged by the block DCB in Fig. 8. As mentioned in Section III-B, the need for Doppler centroid correction depends on the GMTI methodology, and therefore this operation is optional.

\section{Estimation OF PARAmeters AND OFFSETS}

Section IV presents the mathematical framework used for the estimation of important parameters and offsets that are required by the proposed algorithm, such as azimuth antenna patterns, along-track baselines, phase and magnitude offsets, Doppler centroid, and the attitude angle (yaw, pitch, and roll) offsets of the antenna array.

\section{A. Antenna Pattern Estimation}

The envelope of the average two-way diagram of the azimuth antenna pattern can be estimated from the radar data $\mathbf{Z}$ in the range-Doppler domain by averaging all available range bins $K$ for each Doppler frequency bin $f_{\mathrm{a}}$ [9]

$$
A_{\mathrm{m}}\left(f_{\mathrm{a}}, m\right)=\sqrt{\frac{1}{K} \sum_{k=1}^{K}\left|\mathbf{Z}\left(r_{\mathrm{k}}, f_{\mathrm{a}}, m\right)\right|^{2}}, \quad m=1, \ldots, M
$$




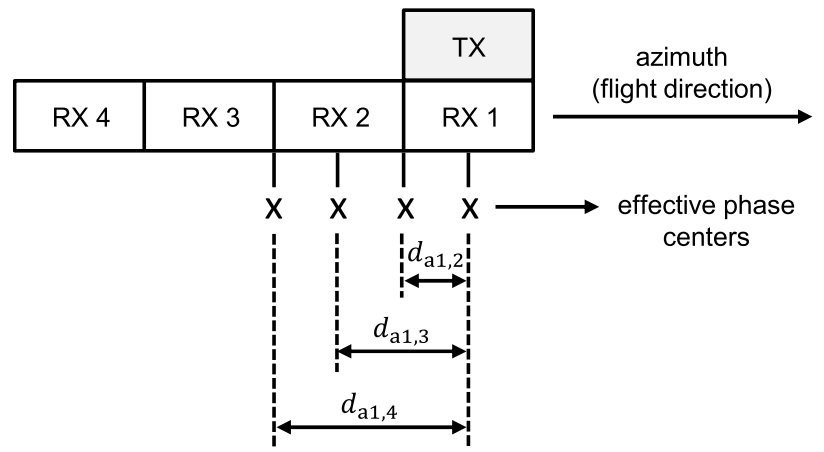

Fig. 9. X-band configuration of DLR's airborne F-SAR: effective alongtrack baselines assuming channel RX1 as reference $\left(\left|d_{\mathrm{a} 1,2}\right|=0.1 \mathrm{~m}\right.$; $\left.\left|d_{\mathrm{a} 1,3}\right|=0.2 \mathrm{~m} ;\left|d_{\mathrm{a} 1,4}\right|=0.3 \mathrm{~m}\right)$.

where $m$ denotes the index of the RX channel (see Fig. 1) and $r_{\mathrm{k}}$ is the range to bin $k$.

Note that the obtained envelope amplitude depends on the clutter reflectivity, which is assumed to be equal for all TX-RX antenna combinations. Although the estimated antenna envelopes are not radiometrically correct, they can be used for the magnitude offset estimation (a radiometric calibration is generally not needed for moving target detection and DOA estimation). In case of high squint angles, a range-dependent Doppler centroid correction (see Section V-B) is required to remove the J-Hook before estimating the azimuth antenna pattern envelopes with (13).

\section{B. Magnitude Offset Estimation}

The magnitude (or gain) offsets with respect to the two-way reference antenna envelope TX-RX1 can be obtained from the azimuth antenna pattern envelope maxima according to

$$
\rho_{1, \mathrm{~m}}=\frac{\max \left(A_{1}\right)}{\max \left(A_{\mathrm{m}}\right)}, \quad m=2, \ldots, M
$$

where channel RX1 is assumed as reference. Although other methods are possible for estimating the magnitude offsets [3], the use of the maxima in (14) is sufficient since the azimuth antenna pattern envelopes are similar (see Fig. 16).

\section{Along-Track Baseline Estimation}

The X-band configuration of DLR's airborne system F-SAR contains four RX channels aligned along the azimuth or flight direction [10], [11]. The physical separation between two adjacent RX antenna centers is given by

$$
b_{\mathrm{a}}=2 \cdot d_{\mathrm{a}}
$$

where $d_{\mathrm{a}}$ denotes the effective along-track baseline.

For the F-SAR, the nominal effective along-track baselines among the RX channels (assuming channel RX1 as reference) are $\left|d_{\mathrm{a} 1,2}\right|=0.1 \mathrm{~m},\left|d_{\mathrm{a} 1,3}\right|=0.2 \mathrm{~m}$, and $\left|d_{\mathrm{a} 1,4}\right|=0.3 \mathrm{~m}$, as depicted in Fig. 9.

The effective along-track baselines $d_{\mathrm{a} 1, \mathrm{~m}}$ can be estimated from the radar data based on the slopes of the ATI phases $\phi_{1, \mathrm{~m}}$ along the Doppler frequency $f_{\mathrm{a}}$ according to [1]

$$
\phi_{1, \mathrm{~m}}\left(f_{\mathrm{a}}\right)=-\frac{d_{\mathrm{a} 1, \mathrm{~m}}}{v_{\mathrm{p}}} \cdot f_{\mathrm{a}}
$$

where $m$ denotes the index of the RX channel (see Fig. 1).

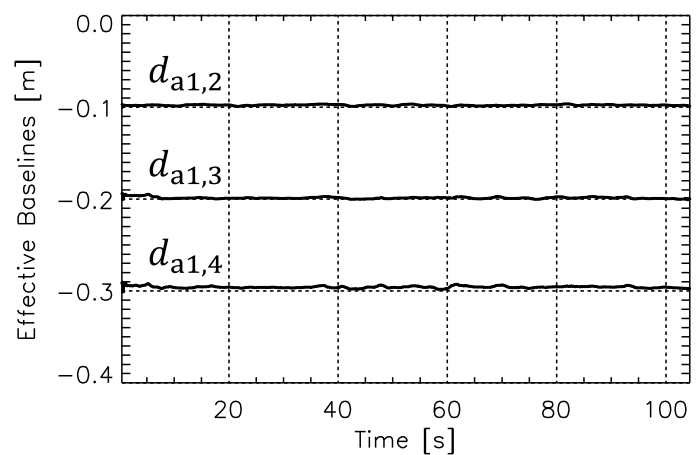

Fig. 10. Effective along-track baselines estimated from data set 2 (see Section VI-A).

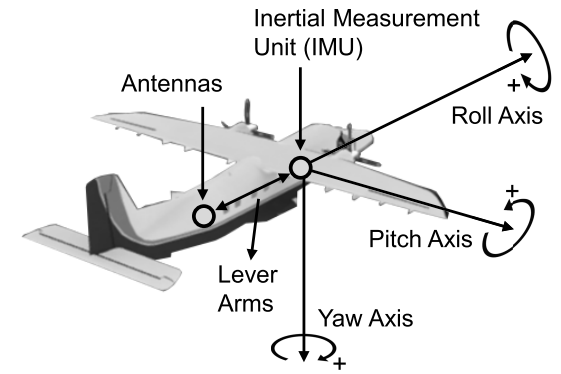

Fig. 11. Axes of the aircraft's attitude angles: yaw, pitch, and roll. The physical distance between the IMU and the antennas is known as "lever arms.".

Fig. 10 shows the effective along-track baselines estimated from data set 2 (see Section VI-A). In this experiment, a moving window was applied for averaging the samples along the azimuth time. The means and standard deviations obtained from the baselines $d_{\mathrm{a} 1,2}, d_{\mathrm{a} 1,3}$, and $d_{\mathrm{a} 1,4}$ were, respectively, $[-0.098,0.001],[-0.199,0.001]$, and $[-0.296,0.002] \mathrm{m}$. From Fig. 5 (in Section II-E), it can be seen that for a baseline estimation error of $\Delta b_{\mathrm{a}}=0.002 \mathrm{~m}$ (which is the highest standard deviation obtained from $d_{\mathrm{a} 1,4}$ ), the expected target azimuth error $\Delta x_{\mathrm{t}}$ is better than $2 \mathrm{~m}$, which is acceptable.

\section{Doppler Centroid Estimation}

Atmospheric turbulences are a serious problem for airborne SAR systems, since they prevent the aircraft from following a straight flight trajectory. The attitude angles of the aircraft play an important role, especially if the platform is equipped with a flat antenna array which does not allow any zero-Doppler beam steering, as in the case of DLR's airborne system F-SAR. For instance, the nominal aircraft pitch angles are often in the order of $1^{\circ}$ or $2^{\circ}$, reaching up to $7^{\circ}$ depending on the type of the aircraft and on the airspeed [17].

The axes of the aircraft's attitude angles are depicted in Fig. 11. The pitch angle is positive when the aircraft's nose points upward and negative when it points downward. The yaw angle is positive when the aircraft's nose points toward the right (with respect to the flight direction) and negative when it points toward the left. The roll angle is positive when the 


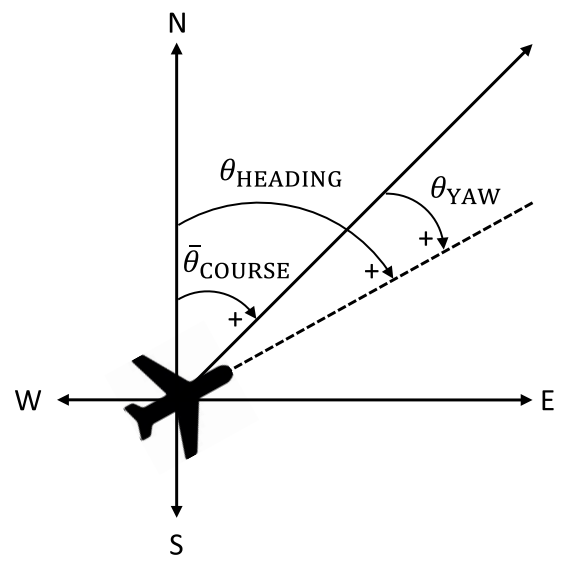

Fig. 12. Top view geometry illustrating the aircraft's yaw angle, heading direction, and mean flight course.

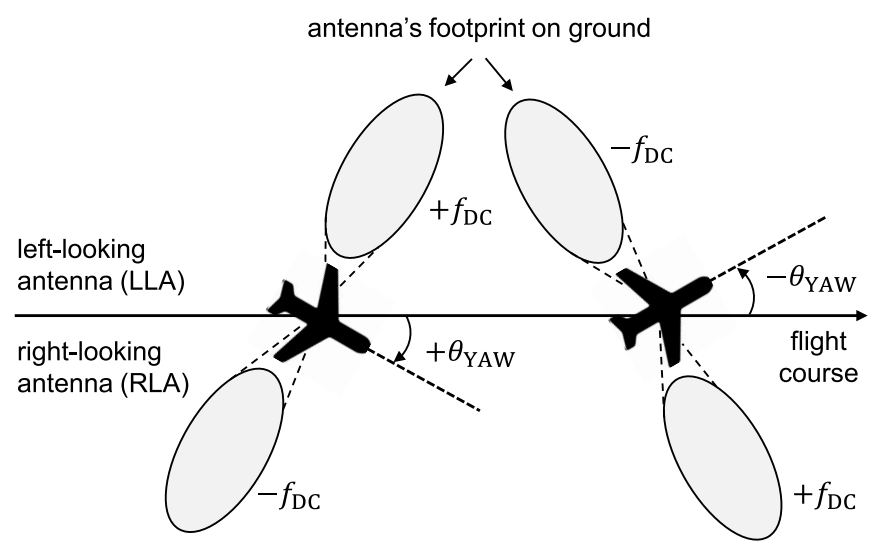

Fig. 13. Top view geometry illustrating the signs of the yaw angle and Doppler centroid for LLA and RLA.

right wing of the aircraft rolls downward and negative when it rolls upward.

The F-SAR's IMU provides the aircraft's pitch, roll, and heading angles with respect to the true North. The aircraft's heading direction (see Fig. 12) can be used for obtaining the yaw angle according to

$$
\theta_{\mathrm{YAW}, \mathrm{IMU}}(t)=\theta_{\mathrm{HEADING}, \mathrm{IMU}}(t)-\bar{\theta}_{\mathrm{COURSE}}
$$

where $\bar{\theta}_{\text {COURSE }}$ denotes the aicraft's mean flight course with respect to the true North, which is obtained according to the GPS coordinates of the aircraft. For instance, the parameter $\bar{\theta}_{\text {COURSE }}$ can be updated for each coherent processing interval (CPI).

The relationship between the yaw angle and the sign of the Doppler centroid is depicted in Fig. 13. Note that for a left-looking antenna (LLA), a positive yaw angle causes a positive Doppler centroid, since the antenna is steered forward with respect to the flight course $\bar{\theta}_{\text {COURSE }}$. For a right-looking antenna (RLA), a positive yaw angle causes a negative Doppler centroid since the antenna is steered backward with respect to the flight course $\bar{\theta}_{\text {COURSE. }}$.

The proposed calibration algorithm requires the information whether the antenna is installed left-looking or right-looking.
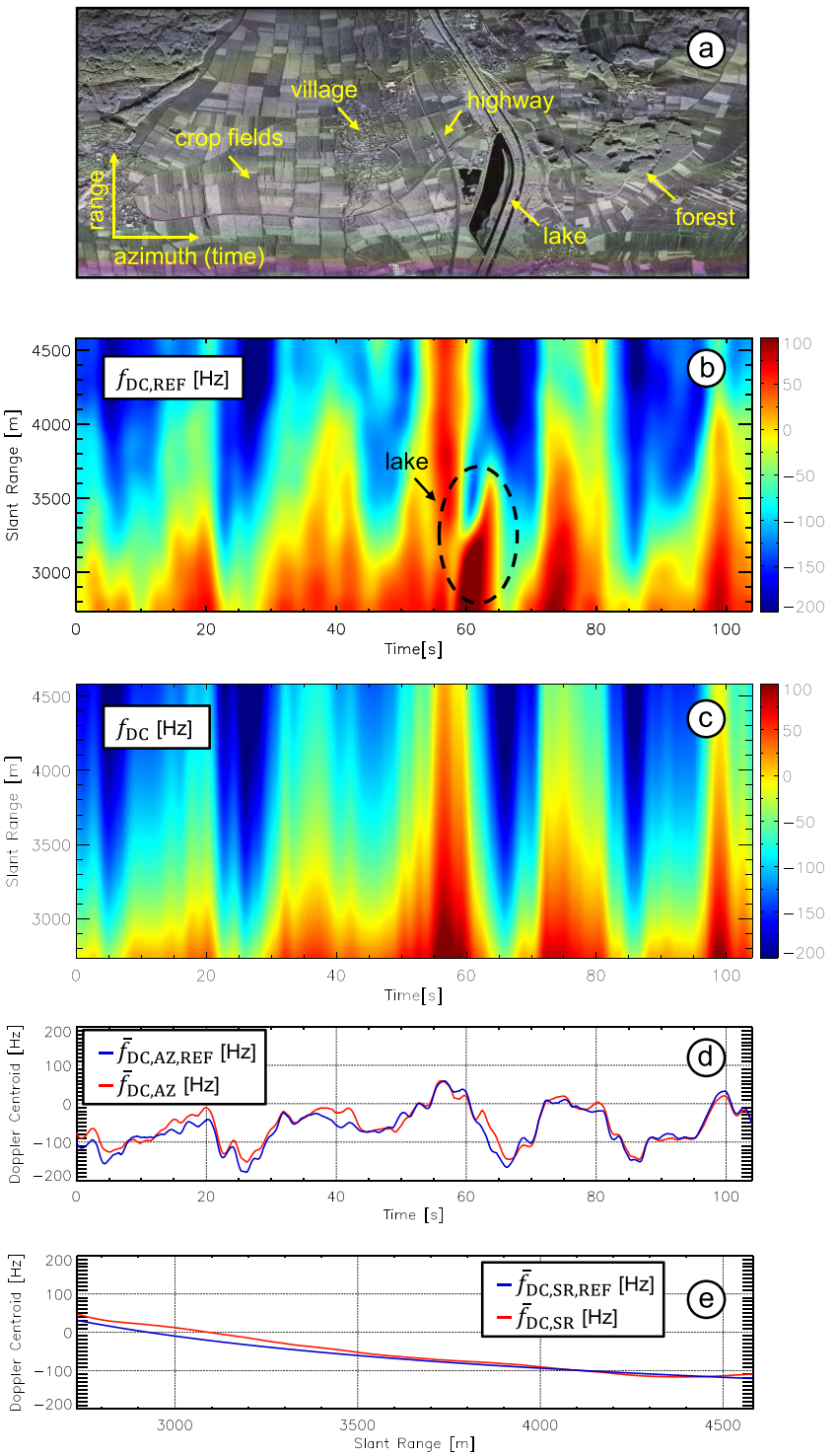

Fig. 14. (a) Focused SAR image from data set 2. (b) Doppler centroid estimated from the radar data (channel RX 1). (c) Doppler centroid computed using the IMU attitude angles and the estimated antenna attitude angle offsets. (d) Doppler centroid profiles over the azimuth time $t$. (e) Doppler centroid profiles over the slant range $r_{\mathrm{k}}$, where the "J-Hook" shape can be clearly recognized from near to far range.

The squint angle $\Psi_{\mathrm{sq}}$ and the clutter Doppler centroid $f_{\mathrm{DC}}$ are generally related as

$$
f_{\mathrm{DC}}=\frac{2 \cdot v_{\mathrm{p}}}{\lambda} \sin \left(\Psi_{\mathrm{sq}}\right) \text {. }
$$

The squint angle $\Psi_{\mathrm{sq}}$ causes a range-dependent Doppler centroid shift, which especially for large squint angles can be clearly recognized in the range-Doppler domain as a J-shaped pattern known as "J-Hook" [16] [see Fig. 14(e)].

The accurate knowledge of the varying Doppler centroid is especially important for the PD STAP technique. Indeed, PD STAP requires Doppler centroid correction to achieve accurate CCM estimation (see the Appendix), which impacts the estimation accuracy of the moving target position and velocity. 
The squint angle variation can be expressed for a LLA and a RLA (see Fig. 13), respectively, as [18]

$$
\begin{aligned}
& \Psi_{\mathrm{sq}, \mathrm{LLA}}\left(r_{\mathrm{k}}, t\right) \\
& \approx \\
& \quad \sin ^{-1}\left[\cos \left(\theta_{\mathrm{i}}\left(r_{\mathrm{k}}\right)+\theta_{\mathrm{ROLL}, \mathrm{ANT}}(t)\right) \cdot \tan \left(\theta_{\mathrm{PITCH}, \mathrm{ANT}}(t)\right)\right. \\
& \left.\quad+\sin \left(\theta_{\mathrm{i}}\left(r_{\mathrm{k}}\right)+\theta_{\mathrm{ROLL}, \mathrm{ANT}}(t)\right) \cdot \tan \left(\theta_{\mathrm{YAW}, \mathrm{ANT}}(t)\right)\right] \\
& \Psi_{\mathrm{Sq}, \mathrm{RLA}}\left(r_{\mathrm{k}}, t\right) \\
& \approx \\
& \sin ^{-1}\left[\cos \left(\theta_{\mathrm{i}}\left(r_{\mathrm{k}}\right)+\theta_{\mathrm{ROLL}, \mathrm{ANT}}(t)\right) \cdot \tan \left(\theta_{\mathrm{PITCH}, \mathrm{ANT}}(t)\right)\right. \\
& \left.\quad-\sin \left(\theta_{\mathrm{i}}\left(r_{\mathrm{k}}\right)+\theta_{\mathrm{ROLL}, \mathrm{ANT}}(t)\right) \cdot \tan \left(\theta_{\mathrm{YAW}, \mathrm{ANT}}(t)\right)\right]
\end{aligned}
$$

where $\theta_{\mathrm{YAW}, \mathrm{ANT}}, \theta_{\mathrm{PITCH}, \mathrm{ANT}}$, and $\theta_{\mathrm{ROLL}, \mathrm{ANT}}$ denote the yaw, pitch, and roll angles of the antenna array, respectively. These angles can be expressed as

$$
\begin{aligned}
\theta_{\mathrm{YAW}, \mathrm{ANT}}(t) & =\theta_{\mathrm{YAW}, \mathrm{IMU}}(t)+\Delta \theta_{\mathrm{YAW}} \\
\theta_{\mathrm{PITCH}, \mathrm{ANT}}(t) & =\theta_{\mathrm{PITCH}, \mathrm{IMU}}(t)+\Delta \theta_{\mathrm{PITCH}} \\
\theta_{\mathrm{ROLL}, \mathrm{ANT}}(t) & =\theta_{\mathrm{ROLL}, \mathrm{IMU}}(t)+\Delta \theta_{\mathrm{ROLL}}
\end{aligned}
$$

where $\theta_{\mathrm{YAW}, \mathrm{IMU}}, \theta_{\mathrm{PITCH}, \mathrm{IMU}}$, and $\theta_{\mathrm{ROLL}, \mathrm{IMU}}$ denote the yaw, pitch, and roll angles of the aircraft, which are obtained from the IMU system. The terms $\Delta \theta_{\mathrm{YAW}}, \Delta \theta_{\mathrm{PITCH}}$, and $\Delta \theta_{\mathrm{ROLL}}$ are the attitude angle offsets that can be estimated by the proposed algorithm (see Section IV-E).

The attitude angle offsets may arise due to an imperfect alignment of the antenna patches or elements, as well as due to the antenna pod's mounting on the aircraft's fuselage (i.e., nonparallel with respect to the aircraft's longitudinal axis).

The Doppler centroid variation can be expressed for the LLA and the RLA (see Fig. 13), respectively, as

$$
\begin{aligned}
& f_{\mathrm{DC}, \mathrm{LLA}}\left(r_{\mathrm{k}}, t\right) \\
& \approx \frac{2 \cdot v_{\mathrm{p}}}{\lambda}\left[\cos \left(\theta_{\mathrm{i}}\left(r_{\mathrm{k}}\right)+\theta_{\mathrm{ROLL}, \mathrm{ANT}}(t)\right) \cdot \tan \left(\theta_{\mathrm{PITCH}, \mathrm{ANT}}(t)\right)\right. \\
& \left.+\sin \left(\theta_{\mathrm{i}}\left(r_{\mathrm{k}}\right)+\theta_{\mathrm{ROLL}, \mathrm{ANT}}(t)\right) \cdot \tan \left(\theta_{\mathrm{YAW}, \mathrm{ANT}}(t)\right)\right] \\
& f_{\mathrm{DC}, \mathrm{RLA}}\left(r_{\mathrm{k}}, t\right) \\
& \approx \frac{2 \cdot v_{\mathrm{p}}}{\lambda}\left[\cos \left(\theta_{\mathrm{i}}\left(r_{\mathrm{k}}\right)+\theta_{\mathrm{ROLL}, \mathrm{ANT}}(t)\right) \cdot \tan \left(\theta_{\mathrm{PITCH}, \mathrm{ANT}}(t)\right)\right. \\
& \left.-\sin \left(\theta_{\mathrm{i}}\left(r_{\mathrm{k}}\right)+\theta_{\mathrm{ROLL}, \mathrm{ANT}}(t)\right) \cdot \tan \left(\theta_{\mathrm{YAW}, \mathrm{ANT}}(t)\right)\right] .
\end{aligned}
$$

The 2-D Doppler centroid estimated according to (24) or (25) is used for the Doppler centroid correction (see Section V-B) in case the PD STAP technique is considered.

In practice, the velocity of the platform $v_{\mathrm{p}}$ may also change slightly over the azimuth time $t$. Therefore, the estimation of the platform's velocity needs to be carried out regularly during the successive CPIs if no computationally time-consuming first-order motion compensation [15] is performed beforehand.

\section{E. Attitude Angle Offset Estimation}

The attitude angle offsets $\boldsymbol{\Delta} \boldsymbol{\theta}=\left(\Delta \theta_{\mathrm{YAW}}, \Delta \theta_{\mathrm{PITCH}}, \Delta \theta_{\mathrm{ROLL}}\right)$ are estimated from the range-compressed data. An example considering data set 2 (see Section VI-A) is shown in Fig. 14, in which the focused SAR image is shown in Fig. 14(a).

The following main steps are required for estimating the attitude angle offsets.

1) The reference Doppler centroid of the scene $f_{\mathrm{DC}, \mathrm{REF}}\left(r_{\mathrm{k}}, t\right)$ is estimated from the reference channel RX1 (e.g., using the energy balancing method proposed in [19]). The result is shown in Fig. 14(b), where a 2-D moving window was applied for selecting data patches for the Doppler centroid estimation.

2) The attitude angle offsets are obtained by means of multidimensional minimization (e.g., using the downhill simplex [20] or Powell's method [21])

$$
\operatorname{argmin}_{\Delta \theta}\left\{\max \left(\left\|f_{\mathrm{DC}, \mathrm{REF}}\left(r_{\mathrm{k}}, t\right)-f_{\mathrm{DC}}\left(r_{\mathrm{k}}, t, \Delta \boldsymbol{\theta}\right)\right\|\right)\right\}
$$

where $f_{\mathrm{DC}, \mathrm{REF}}\left(r_{\mathrm{k}}, t\right)$ is estimated from the radar data and $f_{\mathrm{DC}}\left(r_{\mathrm{k}}, t, \boldsymbol{\Delta} \boldsymbol{\theta}\right)$ can be obtained from (24) or (25) for the LLA and the RLA, respectively. The $\Delta \theta$ dependence, denoted here explicitly, follows (21)-(23).

The attitude angle offsets estimated with (26) for data set 2 were $\Delta \theta_{\mathrm{YAW}}=0.86^{\circ}, \Delta \theta_{\mathrm{PITCH}}=0.54^{\circ}$, and $\Delta \theta_{\mathrm{ROLL}}=-0.95^{\circ}$, which are strictly valid for the reference channel RX1.

Fig. 14(c) shows the Doppler centroid obtained with (24) using the estimated attitude angle offsets. Indeed, the Doppler centroid estimated from the measured radar data [shown in Fig. 14(b)] and the Doppler centroid computed with (24) [shown in Fig. 14(c)] are visually comparable. The main differences between both the images are explained by two factors.

1) The measured data at some spots were nonhomogeneous and contained moving vehicles that were not removed before estimating $f_{\mathrm{DC}, \mathrm{REF}}\left(r_{\mathrm{k}}, t\right)$. As a result, an expected bias was obtained especially in the region of the highway [see Fig. 14(a)] due to the additional Doppler shift introduced by fast vehicles;

2) A bias can be seen in the region of the lake (see dark red region highlighted in Fig. 14(b), where the highest Doppler frequency values were obtained. This bias was expected due to the very low reflectivity of the lake region, which prevented accurate estimates of the Doppler centroid.

Fig. 14(d) shows the average azimuth profiles of the Doppler centroid variation previously shown in Fig. 14(b) and (c). Note in Fig. 14(d) that the average azimuth profiles match very well especially in the regions of crop fields, where the clutter is more homogeneous. In contrast, mismatches are recognized especially in the central part of data set 2 [see Fig. 14(a)]. The average azimuth profiles were obtained according to

$$
\begin{aligned}
\bar{f}_{\mathrm{DC}, \mathrm{AZ}, \mathrm{REF}}(t) & =\frac{1}{K} \sum_{k=0}^{K-1} f_{\mathrm{DC}, \mathrm{REF}}\left(r_{\mathrm{k}}, t\right) \\
\bar{f}_{\mathrm{DC}, \mathrm{AZ}}(t) & =\frac{1}{K} \sum_{k=0}^{K-1} f_{\mathrm{DC}, \mathrm{ATT}}\left(r_{\mathrm{k}}, t\right)
\end{aligned}
$$

where $K$ is the number of available range bins (see Table II).

Fig. 14(e) shows the average slant range profiles, which were obtained according to

$$
\begin{aligned}
\bar{f}_{\mathrm{DC}, \mathrm{SR}, \mathrm{REF}}\left(r_{\mathrm{k}}\right) & =\frac{1}{N_{\mathrm{a}}} \sum_{t=0}^{N_{\mathrm{a}}-1} f_{\mathrm{DC}, \mathrm{REF}}\left(r_{\mathrm{k}}, t\right) \\
\bar{f}_{\mathrm{DC}, \mathrm{SR}}\left(r_{\mathrm{k}}\right) & =\frac{1}{N_{\mathrm{a}}} \sum_{t=0}^{N_{\mathrm{a}}-1} f_{\mathrm{DC}, \mathrm{ATT}}\left(r_{\mathrm{k}}, t\right)
\end{aligned}
$$

where $N_{\mathrm{a}}$ is the number of azimuth samples (see Table II). 


\section{F. Phase Offset Estimation}

The phase offsets may be introduced by the hardware of the radar system (e.g., antenna, connectors, cables with different electrical lengths). The phase offset of each channel $m$ with respect to RX 1 (denoted $\varphi_{1, \mathrm{~m} \text {,offset }}$ ) is assumed to be a constant that can be estimated for the calibration flight and stored for later use. It is a property of the antenna assembly to be updated if, for example, the cables are replaced, and then assumed to be invariant between subsequent flights.

At least the following main steps are needed for estimating the phase offsets $\left(\varphi_{1, \mathrm{~m}, \text { offset }}\right)$ from the radar data.

1) Obtain the radar range-compressed data.

2) Select a data patch containing homogeneous clutter.

3) Estimate the phase offsets in time domain for each pair of RX channels according to [22]

$$
\varphi_{1, \mathrm{~m}, \text { offset }}=\arg \left(\frac{1}{N} \sum_{k=1}^{N} \mathbf{z}_{1}(k) \cdot \mathbf{z}_{\mathrm{m}}^{*}(k)\right)
$$

where $\mathbf{z}$ is the radar data in the time domain, $m$ is the index of the RX channel (see Fig. 1), and $N$ is the total number of samples. It is important to point out that this step requires co-registration in the range and in the azimuth direction, which are generally time-consuming operations.

\section{Correction of Channel Imbalances}

The main steps needed for the channel imbalance correction are presented in Section V. The correction is performed adaptively depending on the IMU attitude angles and the measured range and requires the previously estimated parameters and offsets described in Section IV.

\section{A. Antenna Aperture Switching Correction}

The antenna aperture switching (AS) technique can improve the GMTI performance of the radar systems by creating additional phase centers and larger ATI baselines. Yet, this operation introduces a time delay that needs to be corrected [23].

The antenna AS correction can be carried out in the Doppler frequency domain according to

$$
\mathbf{Z}_{\mathrm{AS}, \text { corr }}\left(r_{\mathrm{k}}, f_{\mathrm{a}}, m\right)=\mathbf{Z}\left(r_{\mathrm{k}}, f_{\mathrm{a}}, m\right) \cdot \exp \left\{-j 2 \pi f_{\mathrm{a}} \Delta t_{\mathrm{AS}}\right\}
$$

where $\mathbf{Z}_{\mathrm{AS} \text {,corr }}$ denotes the radar data in the range-Doppler domain after the antenna AS correction, $\Delta t_{\mathrm{AS}}=1 /(2 \cdot \mathrm{PRF})$ denotes the time lag introduced by the F-SAR antenna AS (which contributes to the effective along-track baseline) [24], and PRF denotes the pulse repetition frequency. It is pointed out that different AS schemes cause different time lags to be corrected [23].

\section{B. Doppler Centroid Correction}

The need for Doppler centroid correction depends on the used GMTI methodology. In this article, the PD STAP technique is used, and thus the Doppler centroid correction is required for obtaining an accurate CCM estimation (see the Appendix), which impacts the moving target's detection performance and the parameter estimation accuracy.
The Doppler centroid can be corrected in the time domain for each range bin $r_{\mathrm{k}}$ and for each RX channel $m$ according to

$$
\mathbf{z}_{\mathrm{FDC}, \text { corr }}\left(r_{\mathrm{k}}, t, m\right)=\mathbf{z}\left(r_{\mathrm{k}}, t, m\right) \cdot \exp \left\{-j 2 \pi t \Delta f_{\mathrm{DC}}\left(r_{\mathrm{k}}\right)\right\}
$$

where $\mathbf{z}_{\mathrm{FDC} \text {,corr }}$ denotes the radar data in the time domain after the Doppler centroid correction. The Doppler centroid variation along the slant range $\Delta f_{\mathrm{DC}}$ can be obtained, for instance, for each CPI according to

$$
\Delta f_{\mathrm{DC}}\left(r_{\mathrm{k}}\right)=\frac{1}{n_{\mathrm{a}, \mathrm{CPI}}} \sum_{t=0}^{n_{\mathrm{a}, \mathrm{CP}-1}} f_{\mathrm{DC}}\left(r_{\mathrm{k}}, t\right)
$$

where $n_{\mathrm{a}, \mathrm{CPI}}$ is the number of azimuth samples contained in a single CPI (e.g., $n_{\mathrm{a}, \mathrm{CPI}}=128$ azimuth samples) and $f_{\mathrm{DC}}\left(r_{\mathrm{k}}, t\right)$ is estimated according to (24) or (25) for the reference channel RX1 [see Fig. 14(c)]. Thus, the range-dependent Doppler centroid is corrected for each CPI independently. Note that in (33) the same Doppler centroid $\Delta f_{\mathrm{DC}}\left(r_{\mathrm{k}}\right)$ is applied to all RX channels.

\section{Phase Correction}

The phase correction (assuming channel RX1 as reference) can be carried out in the time domain according to

$$
\mathbf{z}_{\mathrm{ATI}, \text { corr }}\left(r_{\mathrm{k}}, t, m\right)=\mathbf{z}\left(r_{\mathrm{k}}, t, m\right) \cdot \exp \left\{j \Delta \hat{\varphi}_{1, \mathrm{~m}}\left(r_{\mathrm{k}}, t\right)\right\}
$$

where $\mathbf{z}_{\text {ATI,corr }}$ denotes the radar data in the time domain after the phase correction and $\Delta \hat{\varphi}_{1, m}\left(r_{\mathrm{k}}, t\right)$ denotes the ATI phases estimated between the RX channels according to

$$
\Delta \hat{\varphi}_{1, \mathrm{~m}}\left(r_{\mathrm{k}}, t\right)=\frac{4 \cdot \pi}{\lambda} \cdot\left[d_{\mathrm{a} 1, \mathrm{~m}} \cdot \sin \left(\Psi_{\mathrm{sq}}\left(r_{\mathrm{k}}, t\right)\right)\right]+\varphi_{1, \mathrm{~m}, \mathrm{offset}}
$$

where the squint angle $\Psi_{\mathrm{sq}}\left(r_{\mathrm{k}}, t\right)$ is obtained according to (19) for LLA or (20) for RLA, and the offsets are the constants estimated beforehand (e.g., during the calibration flight). It is important to mention that after estimating the phase offsets with (31), the phase correction itself carried out according to (35) does not require co-registration, which saves processing time.

Exemplarily, Fig. 15 shows intermediate results from the phase correction operation carried out between the channels RX1 and RX3 considering data set 2 [see focused SAR image in Fig. 15(a)]. Fig. 15(b) shows the phases measured from the data after the antenna AS correction and before Doppler centroid correction (see the block diagram in Fig. 7), where the predominant yellow background indicates an offset of about $\varphi_{1,3, \text { offset }}=29^{\circ}$ (see Section IV-F).

Fig. 15(c) shows the phases measured from the data after the Doppler centroid correction, where it can be observed that the phase changes over the slant range and time due to the Doppler centroids [shown in Fig. 14(c)] that were applied to the data for Doppler centroid correction.

The phases shown in Fig. 15(c) are corrected using the theoretical phases shown in Fig. 15(d), which are estimated according to (36) using the squint angles obtained from (19). Indeed, 

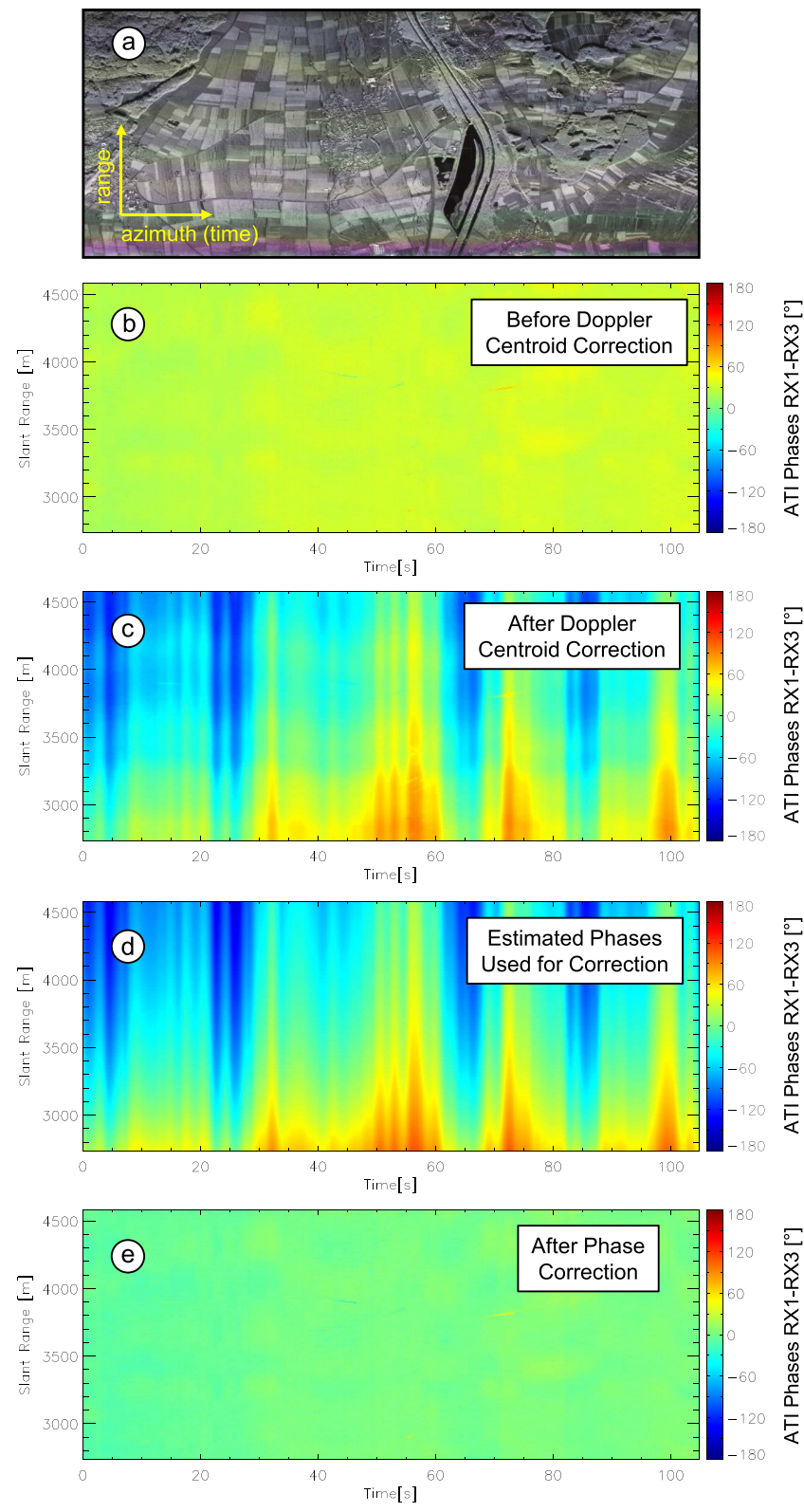

Fig. 15. Intermediate results of the phase correction operation (see main calibration steps in Fig. 7) considering the channels RX1 and RX3. (a) Focused SAR image from data set 2. (b) Phases measured from the data before the Doppler centroid correction. (c) Phases measured from the data after the Doppler centroid correction. (d) Theoretical phases estimated according to (36), which are applied for the phase correction. (e) Phases measured from the data after the phase correction.

it can be noticed that the phases shown in Fig. 15(c) and (d) are visually similar. This indicates that the attitude angles obtained from the IMU and the estimated phase offsets are accurate.

Fig. 15(e) shows the phases measured from the data after the phase correction was carried out according to (35), where the green background indicates that the remaining phase offsets (i.e., $\varphi_{1,3, \text { offset }}=0^{\circ}$ ) are around zero over the entire range and the azimuth time.

In the example shown in Fig. 15, the baseline between RX1 and RX3 was $\left|d_{\mathrm{a} 1,3}\right|=0.2 \mathrm{~m}$ (see Section IV-C).
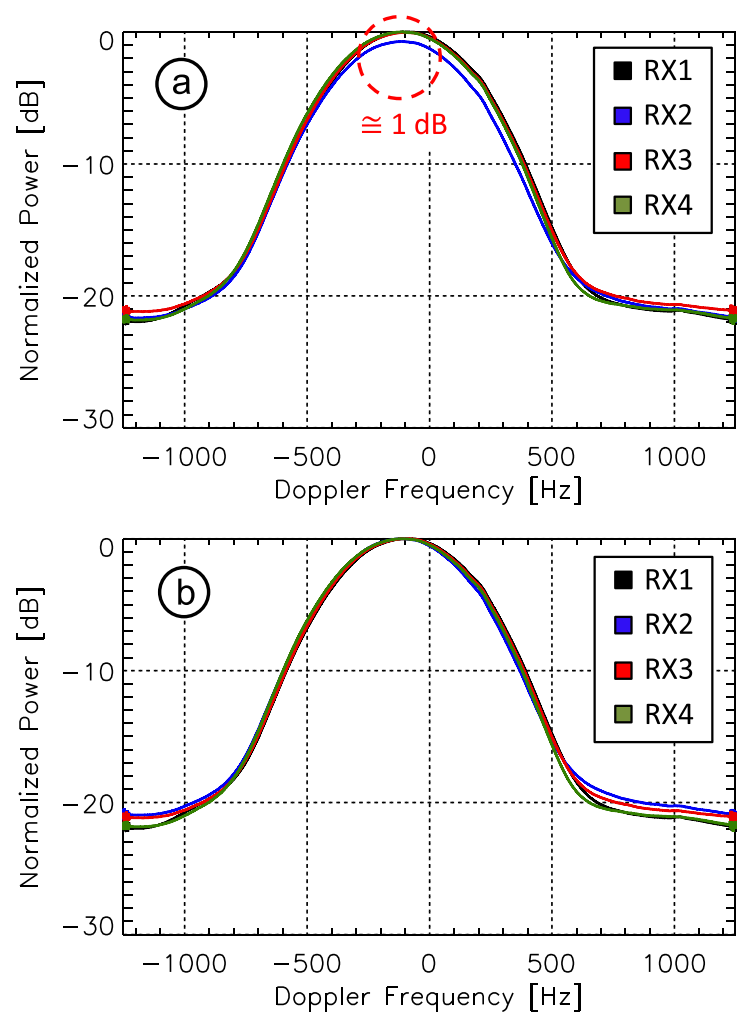

Fig. 16. Normalized azimuth antenna pattern envelopes obtained from homogeneous training data selected from data set 2. (a) Before and (b) after the magnitude correction.

\section{Magnitude Correction}

The magnitude offset correction with respect to channel RX1 can be carried out in the time domain according to

$$
\mathbf{z}_{\mathrm{mag}, \mathrm{corr}}\left(r_{\mathrm{k}}, t, m\right)=\mathbf{z}\left(r_{\mathrm{k}}, t, m\right) \cdot \rho_{1, \mathrm{~m}}
$$

where $\mathbf{z}_{\mathrm{mag} \text {,corr }}$ denotes the radar data in the time domain after the magnitude correction and $\rho_{1, \mathrm{~m}}$ denotes the magnitude offsets previously estimated (see Section IV-B).

Exemplarily, Fig. 16 shows the normalized azimuth antenna pattern envelopes in the Doppler frequency domain estimated according to (13) before and after the magnitude correction. In this example, a homogeneous data patch containing $2048 \times 16384$ range-azimuth samples was obtained from data set 2 (see Section VI-A and Table II). The azimuth antenna patterns were centered at $f_{\mathrm{DC}}=-90.18 \mathrm{~Hz}$ and an offset in the order of $1 \mathrm{~dB}$ can be observed in Fig. 16(a) before magnitude correction. The following magnitude offsets were estimated according to (10): $\rho_{1,2}=1.08, \rho_{1,3}=1.01$ and $\rho_{1,4}=1.05$.

The azimuth antenna pattern envelopes obtained after the magnitude correction [see Fig. 16(b)] can then be applied on the moving target signal model expressed in (1) (i.e., in the beamforming vector to achieve improved DOA angle estimation results).

\section{EXPERIMENTAL RESUlTS}

The proposed algorithm is tested considering two real data sets (see Section VI-A) and is compared with the 


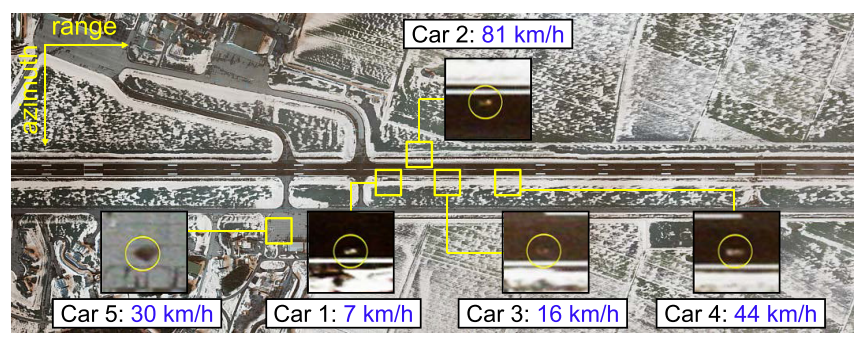

Fig. 17. Optical image of data set 1: Memmingen's Allgäu airport and five cars with controlled movements and speeds.

state-of-the-art DCB technique in terms of processing time (see Section VI-B) and in terms of phase correction accuracy (see Section VI-C). Moreover, the GMTI results are presented and discussed (see Section IV-D) in the frame of traffic monitoring using our PD STAP processor [25]. This last experiment includes a quantitative comparison between the proposed algorithm and the DCB (e.g., number of true and false detections) as well as an evaluation of velocities and signal-to-clutter plus noise ratio (SCNR) of the detections.

\section{A. Experimental Setup and Data Sets}

The multichannel X-band radar data sets were acquired with DLR's airborne system F-SAR [10], [11]. The flight campaign was conducted in the Memmingen area of Germany in February 2007. The polarization of the data sets is "HH."

The antenna pod was mounted at the bottom of F-SAR's fuselage together with a camera, so that optical images could be obtained simultaneously with the radar data [26].

The optical image of data set 1 is shown in Fig. 17, where the runway of the Memmingen's Allgäu airport was located at the broadside direction of the platform and five cars with controlled movement and speed were considered. The cars 1,2, and 4 were equipped with radar reflectors to enhance their RCS, and car 4 had a differential GPS (DGPS) receiver for retrieving reliable geographical reference positions and velocities. Finally, it has to be mentioned that cars 1-4 moved on the edges of the airport's runway and car 5 moved in circles on a nearby parking area.

Data set 2 contains different regions with crop fields (homogeneous clutter), forests and villages (heterogeneous clutter), as well as highways and residential roads with real traffic, as highlighted in Fig. 14(a).

The radar and geometry parameters can be seen in Table I for both the data sets. The effective PRF per RX channel was $2.5 \mathrm{kHz}$. The beamformers were applied using DOA angle steps of $0.05^{\circ}$ within an interval determined by the azimuth antenna beamwidth. In the constant false alarm rate (CFAR) detector, the probability of false alarm was set to $P_{\mathrm{fa}}=10^{-6}$.

The combination of high pitch and yaw angles with a fast-varied terrain would be a very challenging scenario for moving target detection and would require the compensation of the phases induced by a potential across-track baseline [27]. Nevertheless, for the data sets considered in this article, the maximum observed pitch angle is in the order of $\pm 2^{\circ}$ and
TABLE I

RADAR AND GEOMETRY PARAMETERS

\begin{tabular}{lcc}
\hline \hline \multicolumn{1}{c}{ Quantity } & Symbol & Value \\
\hline Speed of light & $\mathrm{c}$ & $2.9979 \times 10^{8} \mathrm{~m} / \mathrm{s}$ \\
Velocity of the platform & $v_{\mathrm{p}}$ & $90 \mathrm{~m} / \mathrm{s}$ \\
Number of receive channels & $M$ & 4 \\
Pulse repetition frequency & PRF & $2500 \mathrm{~Hz}$ \\
Range bandwidth & $f_{\mathrm{r}}$ & $100 \mathrm{MHz}$ \\
Effective along-track baseline & $d_{\mathrm{a}}$ & $0.1 \mathrm{~m}$ \\
Wavelength & $\lambda$ & $0.03125 \mathrm{~m}$ \\
Transmit antenna length & $L_{\mathrm{a}}$ & $0.3 \mathrm{~m}$ \\
Incidence angle range (data set 1) & $\theta_{\mathrm{i}}$ & $25^{\circ}$ to $53^{\circ}$ \\
Incidence angle range (data set 2) & $\theta_{\mathrm{i}}$ & $33^{\circ}$ to $60^{\circ}$ \\
Altitude of the platform (data set 1) & $h$ & $2211 \mathrm{~m}$ \\
Altitude of the platform (data set 2) & $h$ & $2258 \mathrm{~m}$ \\
\hline \hline
\end{tabular}

TABLE II

Number of S AMPLES AND ILLUMINATED SCENE DimENSIONS OF DATA SETS 1 AND 2

\begin{tabular}{lccc}
\hline \hline \multicolumn{1}{c}{ Data Set } & 1 & 2 & 2 (Patch) \\
\hline Azimuth samples $\left(n_{\mathrm{a}}\right)$ & 16384 & 262144 & 16384 \\
Range samples $\left(n_{\mathrm{r}}\right)$ & 1024 & 2048 & 2048 \\
\hline Size in azimuth $(\mathrm{m})$ & 596 & 9361 & 584 \\
Size in slant range $(\mathrm{m})$ & 1226 & 2454 & 2454 \\
Size in ground range $(\mathrm{m})$ & 1917 & 3485 & 3485 \\
\hline \hline
\end{tabular}

the maximum yaw angle is in the order of $\pm 5^{\circ}$. In addition, the data sets were acquired over a moderately flat area without significant terrain elevation change. Thus, the terrain elevation change and the contribution of such comparatively small pitch and yaw angles to the across-track baseline (which impacts the phase estimation and thus the target's azimuth position accuracy) are expected to be minor.

The numbers of samples of data sets 1 and 2 (including the homogeneous training data patch obtained from data set 2) are shown in Table II together with the approximated dimensions of the illuminated scenes. A detailed experiment description of the flight campaign is given in [24] and [26].

The parameters and the offsets required for calibration are estimated once from homogeneous training data (i.e., the data patch obtained from data set 2) and applied to data sets 1 and 2 for the experiments presented in the remainder of Section VI.

\section{B. Processing Time Comparison}

This experiment was carried out by taking into account the processing time required by the blocks phase correction and magnitude correction (see Fig. 7) and by the block DCB (see Fig. 8). Indeed, the processing time required by the blocks AS correction and Doppler centroid correction was not considered since they are common to both the processing chains. 

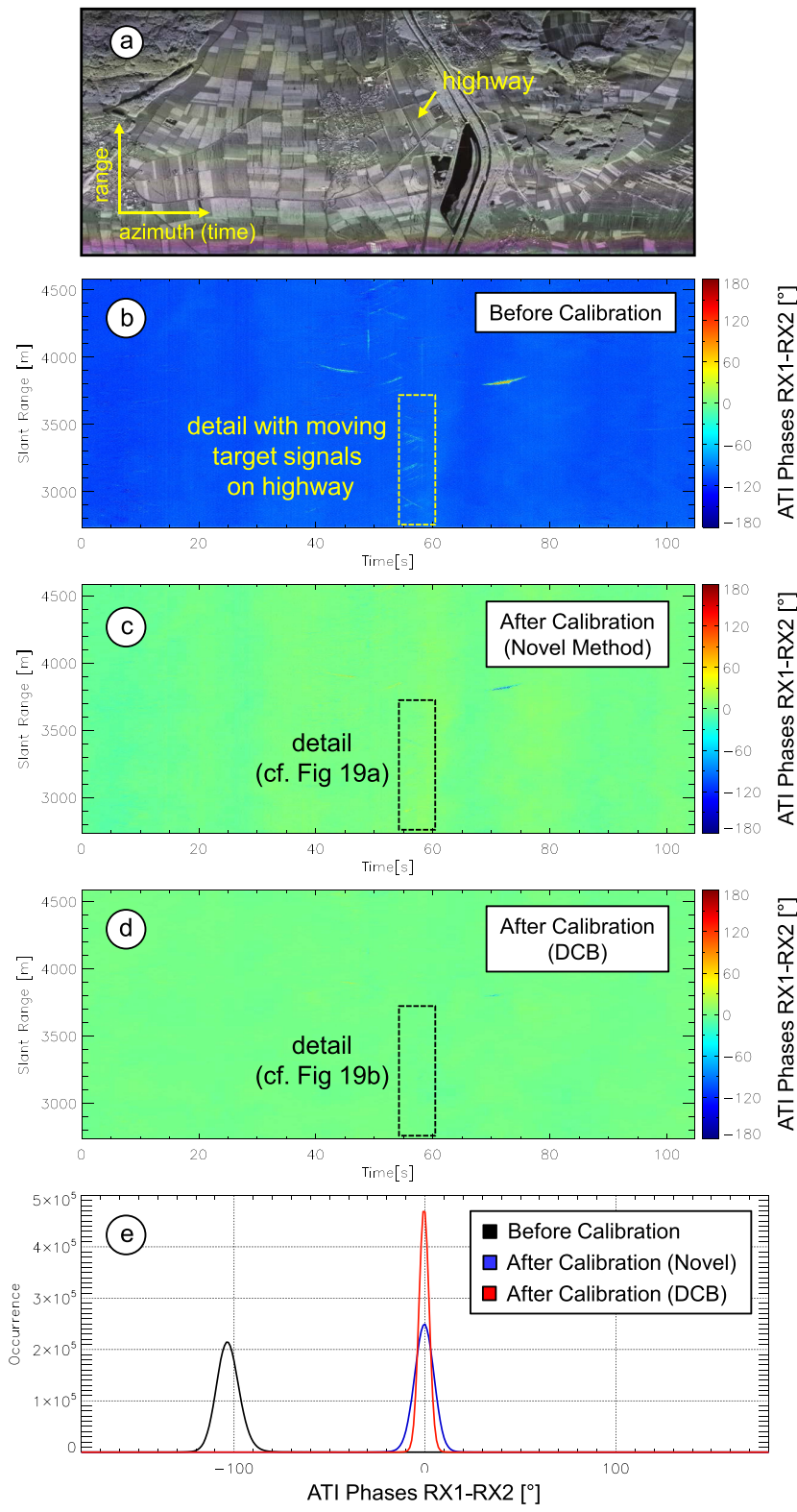

Fig. 18. ATI phases obtained between channels RX1 and RX2. (a) Focused SAR image from data set 2. (b) ATI phases before calibration. (c) ATI phases after calibration using the proposed algorithm. (d) ATI phases after calibration using the state-of-the-art DCB. (e) ATI phase histograms.

Data set 1 and a data patch obtained from data set 2 were processed 1000 times for this experiment. For both the data sets, the proposed algorithm was about 15 times faster than the state-of-the-art DCB. Indeed, the proposed calibration algorithm performs the phase correction based on (35), where all the terms are known or previously estimated (see Section IV). As a result, significantly shorter processing time is required compared with the DCB. Absolute processing times are not considered here, since they severely depend on the used computer architecture and the programming language.

The processing time reduction by a factor of 15 highlights the potential of the proposed algorithm toward real-time applications. For instance, the proposed calibration algorithm can be applied to our fast PD STAP traffic monitoring processor
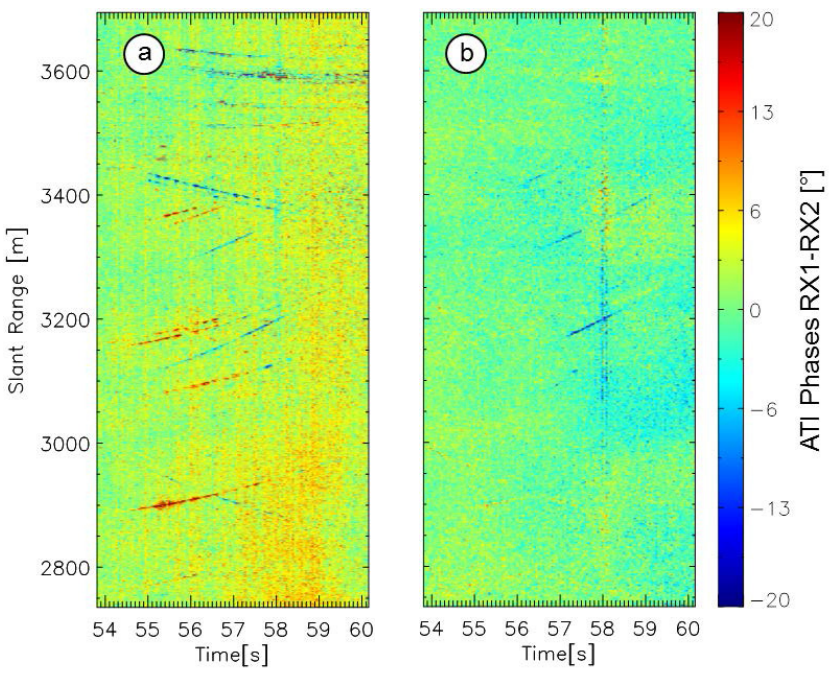

Fig. 19. Detail of the ATI phases obtained from moving target signals using (a) proposed method [see detail in Fig. 18(c)] and (b) DCB technique [see detail in Fig. 18(d)].

with a priori knowledge information presented in [28]. It is pointed out that to achieve real-time processing capabilities, an efficient parallel implementation taking into account the potential multicore and multiprocessor architectures as well as graphical processing units (GPUs) is required. However, this is out of the scope of this article.

\section{Phase Correction Accuracy}

The proposed algorithm and the DCB (see flowcharts shown in Figs. 7 and 8) were compared in terms of phase correction accuracy. Data set 2 was considered for this evaluation since it contains regions with homogeneous and heterogeneous ground clutter, and also moving target signals [see Fig. 18(a)].

Fig. 18(b) shows the ATI phases obtained between channels RX1 and RX2 before calibration. The dashed box shows some moving target signals that can be observed at the central part of Fig. 18(b), where a highway was located at the broadside direction of the radar [see also Fig. 18(a)]. The predominant blue background corresponds to the phase offset $\varphi_{1,2, \text { offset }}=-103^{\circ}$.

The phase offsets estimated between the other pairs of RX channels are $\varphi_{1,3 \text {,offset }}=29^{\circ}$ and $\varphi_{1,4 \text {,offset }}=54^{\circ}$ (not shown in Fig. 18). All offsets were estimated from a homogeneous training data patch obtained from data set 2 .

Fig. 18(c) shows the ATI phases obtained between channels RX1 and RX2 after calibration using the proposed algorithm. The predominant green background indicates that the phase offset was corrected so that the desired ATI phases are centered around zero degrees (i.e., $\varphi_{1,2 \text {,offset }} \cong 0^{\circ}$ ). Similar ATI phases were obtained after calibration using the state-of-the-art DCB technique, as shown in Fig. 18(d).

Fig. 19 shows the region limited by the dashed boxes in Fig. 18(c) and (d), where the ATI phases of the moving target signals can be clearly observed using a different scale $\left[-20^{\circ}, 20^{\circ}\right]$ of the color bar. Fig. 19(a) shows the ATI phases obtained with the proposed algorithm, in which several moving 

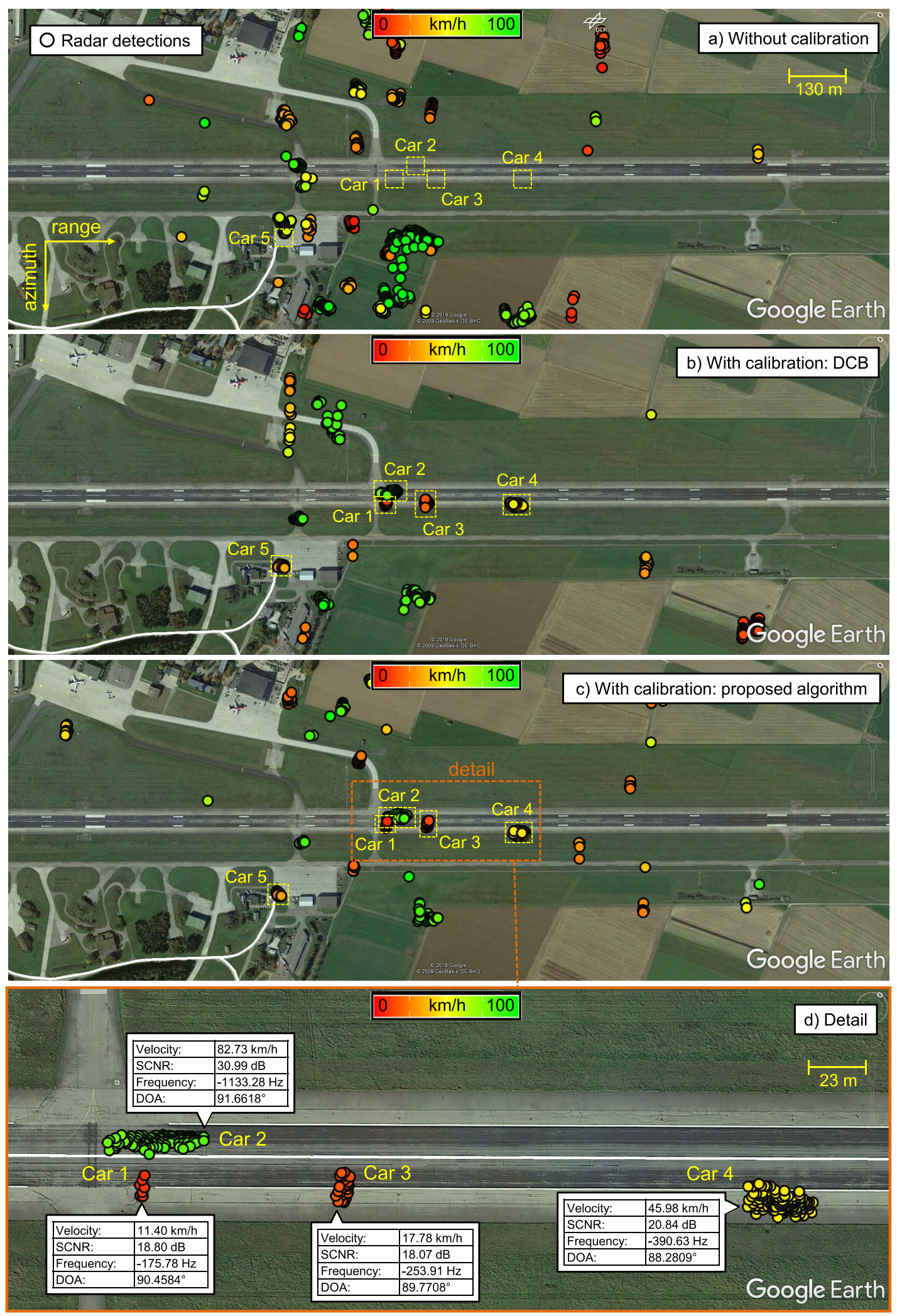

Fig. 20. Google Earth images overlaid with radar detections (circles). Data set 1 was processed. (a) Without calibration. (b) With calibration using the state-of-the-art DCB. (c) With calibration using the proposed algorithm. (d) Detections obtained from cars 1 to 4 .

target signals can be observed. Fig. 19(b) shows the ATI phases obtained with the DCB technique, in which less moving target signals can be seen in comparison to the proposed algorithm [see Fig. 19(a)]. It has to be mentioned that the DCB technique was applied for each CPI independently. Thus, for CPIs that contained several moving targets (e.g., in the region shown 
in detail in Fig. 19), the DCB introduces some target selfwhitening, which explains the reduced number of moving target signals in Fig. 19(b).

Fig. 18(e) shows the histograms of the ATI phases shown in Fig. 18(b)-(d). The histogram shown in black was obtained before calibration [see Fig. 18(b)]. It appears shifted by the phase offset $\left(\varphi_{1,2, \text { offset }}=-103^{\circ}\right)$ and slightly skewed, which are typical effects of the uncalibrated data [1]. The histogram shown in blue was obtained after calibration using the proposed algorithm [see Fig. 18(c)], where the distribution of the ATI phases appears centered around zero degrees. The histogram shown in red was obtained after calibration using the state-of-the-art DCB technique [see Fig. 18(d)]. The ATI phase distribution obtained with the DCB technique was not only centered around zero degrees, but it also presented lower variance in comparison to the proposed algorithm, which indicates better phase correction accuracy.

Indeed, it was expected that the DCB technique would achieve superior phase correction accuracy due to its very sophisticated (and computationally expensive) phase correction operation that is carried out adaptively in the 2-D frequency domain, as verified in [3].

\section{GMTI Results Using Post-Doppler STAP}

The proposed algorithm and the DCB technique are compared for traffic monitoring applications, where both the algorithms are applied on our PD STAP processor [25].

Data set 1 was considered for this experiment since it contained five controlled cars with different movements and speeds. Fig. 20 shows the GMTI results, where the Google Earth images are overlaid with radar detections (circles) obtained from the PD STAP processor. The colors of the radar detections are related to their absolute ground range velocities.

Fig. 20(a) shows the results obtained without calibration. In this case, the phase and magnitude offsets as well as the Doppler centroid were not corrected, resulting in systematic phase errors that extended along the range and the azimuth. Indeed, it is expected that the calculation and application of the CFAR detection thresholds based on the clutter models presented in [23], [29], and [30] would fail, resulting in a number of false detections. Furthermore, the cars cannot be detected and incorrect position and velocity estimates are obtained. Clearly, Fig. 20(a) shows that the PD STAP performance is not acceptable without calibration.

Fig. 20(b) and (c) show the GMTI results obtained by applying the DCB and the proposed algorithm, respectively. It can be seen in these figures that all cars were detected several times in successive CPIs and also fewer false detections were obtained.

Fig. 20(d) shows in detail the radar detections obtained from cars 1 to 4 by applying the proposed algorithm. From Fig. 20(d), it can be seen that the following holds.

1) The slow cars 1 and 3 presented the highest positioning error along the azimuth, while the fastest car 2 presented the lowest. Indeed, slow targets generally present low SCNR, which increases the azimuth displacement error [25]. In contrast, the fastest car 2 presented the
TABLE III

Number of Detections Obtained From Data Set 1

\begin{tabular}{lccc}
\hline \hline Algorithm & All & $\begin{array}{c}\text { True } \\
(\# 1-5)\end{array}$ & $\begin{array}{c}\hat{P}_{\mathrm{fa}} \\
{\left[\times 10^{-6}\right]}\end{array}$ \\
\hline Proposed & 661 & 484 & 10.55 \\
DCB & 575 & 453 & 7.27 \\
\hline \hline
\end{tabular}

lowest azimuth displacement error and can be clearly seen overtaking car 1 in a straight line.

2) Several detections in successive CPIs were obtained for all cars, which shows the potential of the PD STAP processor [25] for traffic monitoring applications. It is pointed out that the clustering and tracking algorithms could be further applied for refining the GMTI results.

3) The information boxes show examples of parameters that can be displayed for detections, such as absolute ground range velocity, Doppler frequency, SCNR, DOA angle (measured with respect to the antenna array axis), among others.

4) The illumination time within the $3-\mathrm{dB}$ azimuth antenna beamwidth was approximately $2 \mathrm{~s}$. During this time, the approximated distances that cars 1-4 moved in the range direction were $[6,46,10,25] \mathrm{m}$, respectively. These distances can be verified visually according to the scale shown in the top-right corner of Fig. 20(d).

A quantitative comparison between the proposed algorithm and the DCB is shown in Table III, which summarizes the following.

1) The number of all radar detections.

2) The number of true detections obtained from cars 1 to 5 (i.e., True $\# 1-5$ ), which can be counted since the positions and velocities of these cars are known for data set 1 .

3) The estimated probability of false alarm $\hat{P}_{\text {fa }}$, which is shown with respect to the desired probability of false alarm of the CFAR detector (i.e., $\hat{P}_{\mathrm{fa}}=10^{-6}$ ).

$\hat{P}_{\text {fa }}$ was estimated according to

$$
\hat{P}_{\mathrm{fa}}=\frac{\text { All-True }_{\# 1-5}}{n_{\mathrm{a}} \cdot n_{\mathrm{r}}}
$$

where $n_{\mathrm{a}}=16384$ and $n_{\mathrm{r}}=1024$ for data set 1 (see Table II).

It can be seen from Fig. 20 and Table III that more true detections are obtained with the proposed algorithm. Yet, the estimated probability of false alarm is higher, which indicates that the DCB obtains less false detections.

Although the proposed algorithm obtained more false detections than the DCB, it is pointed out that most of the false detections can be discarded with our PD STAP processor with a priori knowledge information [25], [28], which uses a road database obtained from the OpenStreetMap [31] and a digital elevation model obtained from the Shuttle Radar Topography Mission [32].

Fig. 21 shows the ground range velocity histograms of the true detections obtained from cars 1 to 5 when applying the proposed algorithm (white bars) and the DCB (black bars). The overlapped areas of the histograms are shown in gray. 


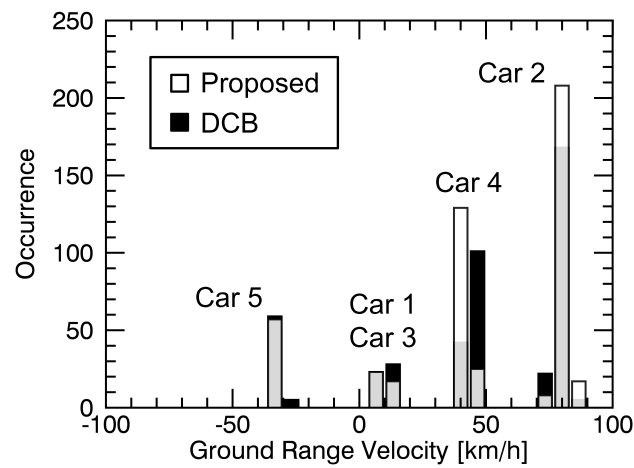

Fig. 21. Ground range velocity histograms of the true detections obtained with the proposed calibration algorithm (white bars) and the DCB (black bars) considering data set 1 . The overlapped areas of the histograms are shown in gray.

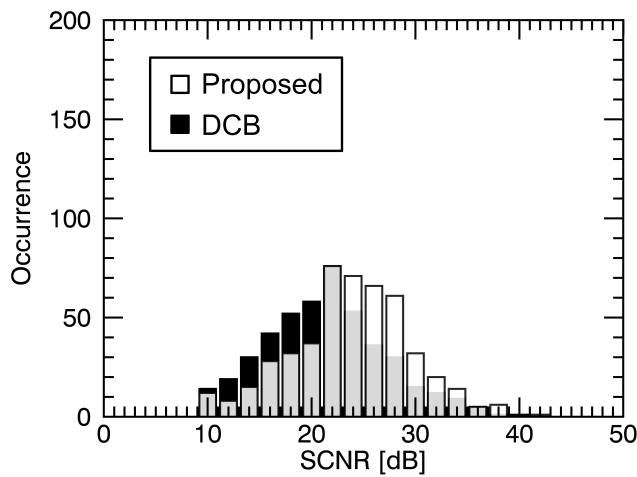

Fig. 22. Histograms of the SCNR of the true detections obtained with the proposed calibration algorithm (white bars) and the DCB (black bars) considering data set 1 . The overlapped areas of the histograms are shown in gray.

It can be seen in Fig. 21 that the ground range velocity estimates obtained with both the calibration algorithms were similar and accurate, according to the ground truth in [26] (see Fig. 17).

Fig. 22 shows the SCNR histograms of the true detections obtained from cars 1 to 5 when applying the proposed algorithm (white bars) and the DCB (black bars). The overlapped areas of the histograms are shown in gray.

It can be seen in Fig. 22 that the mean SCNR of the true detections was similar for both the algorithms (i.e., about $24 \mathrm{~dB})$. The DCB was able to obtain more detections with low SCNR (from 10 to $20 \mathrm{~dB}$ ), which indicates better clutter suppression capability. In contrast, the proposed algorithm obtained more detections with high SCNR (from 24 to $38 \mathrm{~dB}$ ), which can be explained by its robustness against the target self-whitening.

It has to be mentioned that a threshold was applied for both the calibration algorithms to reject detections with SCNRs lower than $10 \mathrm{~dB}$ [33].

\section{CONCLUSION}

This article presents a fast and efficient calibration algorithm for the along-track multichannel systems, which is tested based on real multichannel X-band radar data acquired with DLR's airborne system F-SAR and compared with the state-of-the-art DCB technique.
It is shown that the proposed algorithm is about 15 times faster than the DCB, which highlights its potential toward real-time applications. Similar and accurate velocity estimates are obtained for both the algorithms, whereas the proposed algorithm obtains more true detections and with higher SCNR. On the other hand, the DCB achieves better phase correction accuracy, which results in less false detections.

For traffic monitoring applications, the increased number of false detections obtained by the proposed calibration algorithm can be circumvented to a certain degree with our a priori knowledge-based PD STAP processor [25], where most of the false detections that lie far from the roads are discarded.

The proposed calibration algorithm is also promising for maritime applications, for which it is foreseen that the required parameters and offsets are estimated at the beginning of the flight campaign using homogeneous training data (land clutter) of a neighboring area. In this sense, future experiments on ship detection are foreseen with DLR's new airborne digitalbeamforming system DBF-SAR, which features $12 \mathrm{RX}$ and 4 TX channels.

\section{APPENDIX}

STAP is a class of linear adaptive filtering techniques that operates on space-time observations for enhancing the moving target detection [14]. Several STAP techniques are available in the literature (see [34]-[36]).

For instance, the PD STAP is considered in this article for evaluating the proposed calibration algorithm in terms of the GMTI results. PD STAP is a reduced dimension algorithm that requires less sample support and less processing effort than the classical joint-domain STAP. Besides, similar to other state-of-the-art STAP techniques, PD STAP is able to perform clutter suppression as well as the moving target's detection and parameter estimation (e.g., Doppler frequency, line-ofsight velocity, and DOA angle) [14].

The moving target detection is carried out by applying the adaptive matched filter (AMF) test [37]

$$
\bar{T}\left(r_{\mathrm{k}}, f_{\mathrm{a}}\right)=\frac{\left|\boldsymbol{d}^{H}\left(u_{\text {array }}, f_{\mathrm{a}}\right) \cdot \hat{R}_{\mathrm{W}}^{-1}\left(f_{\mathrm{a}}\right) \cdot \boldsymbol{Z}\left(r_{\mathrm{k}}, f_{\mathrm{a}}\right)\right|^{2}}{\boldsymbol{d}^{H}\left(u_{\text {array }}, f_{\mathrm{a}}\right) \cdot \hat{R}_{\mathrm{W}}^{-1}\left(f_{\mathrm{a}}\right) \cdot \boldsymbol{d}\left(u_{\text {array }}, f_{\mathrm{a}}\right)} \lessgtr \eta
$$

where $[\cdot]^{H}$ denotes the Hermitian operator (complex conjugate transposition) and $\eta$ denotes the CFAR threshold, whose computation is carried out according to the heterogeneous clutter model introduced in [29] and further investigated in [23] and [30]. This clutter model covers different types of heterogeneity, including completely homogeneous clutter. Therefore, it is very flexible for GMTI applications over land. The heterogeneous model includes a texture parameter that is estimated from the training data and describes the degree of heterogeneity of the underlying terrain. For instance, the texture parameter variation for data set 2 is presented in [38].

The normalization in (39) is necessary for providing the desired CFAR behavior [37]. In addition, the term $\boldsymbol{d}$ denotes the beamforming or DOA vector expressed in (1) and the term $\hat{\boldsymbol{R}}_{\mathrm{W}}$ denotes the estimated CCM.

The CCM can be estimated empirically from the training data by applying the sample matrix inverse (SMI) 
technique [39], which is the basis for most modern STAP algorithms (note that other CCM estimation strategies are also possible [40])

$$
\hat{\boldsymbol{R}}_{\mathrm{W}}\left(f_{\mathrm{a}}\right)=\frac{1}{K} \sum_{k=1}^{K} \mathbf{Z}\left(r_{\mathrm{k}}, f_{\mathrm{a}}\right) \cdot \mathbf{Z}^{H}\left(r_{\mathrm{k}}, f_{\mathrm{a}}\right)
$$

where $K$ range bins are used for averaging and $\mathbf{Z}\left(r_{\mathrm{k}}, f_{\mathrm{a}}\right)$ denotes the multichannel data in the range-Doppler domain.

For accurate CCM estimation, it is essential that the multichannel vectors $\mathbf{Z}$ are free of strong discrete scatterers and moving target signals. This condition needs to be ensured by a proper training data selection algorithm (some strategies are presented and compared in [38]). Otherwise, the clutter suppression performance degrades and the moving target signals can be attenuated or canceled. This effect is known as target "self-whitening."

\section{ACKNOWLEDGMENT}

The authors would like to thank the anonymous reviewers for their valuable comments and suggestions that improved significantly both the clarity and the precision of this article.

\section{REFERENCES}

[1] C. H. Gierull, "Digital channel balancing of along-track interferometric SAR data," Defence R\&D Canada, Ottawa, ON, Canada, Tech. Memorandum DRDC Ottawa TM 2003-024, Mar. 2003.

[2] J. H. G. Ender, "The airborne experimental multi-channel SAR-system AER-II," in Proc. Eur. Conf. Synth. Aperture Radar (EUSAR), 1996, pp. $49-52$

[3] A. Bertetich, "Investigation of multi-channel SAR calibration methods for real-time traffic monitoring," M.S. thesis, Dept. Inf. Eng. Comput. Sci., Univ. Trento, Trento, Italy, 2010.

[4] R. M. Goldstein and H. A. Zebker, "Interferometric radar measurement of ocean surface currents," Nature, vol. 328, no. 6132, pp. 707-709, Aug. 1987.

[5] J. F. R. Dickey and M. M. Santa, "Final report on anticlutter techniques," Gen. Electr. Company, Boston, MA, USA, Tech. Rep. R65EMH37, 1953.

[6] J. H. G. Ender, "Space-time processing for multichannel synthetic aperture radar," Electron. Commun. Eng. J., vol. 11, no. 1, pp. 29-38, Feb. 1999

[7] M. Jäger, R. Scheiber, and A. Reigber, "Robust, model-based external calibration of multi-channel airborne SAR sensors using range compressed raw data," Remote Sens., vol. 11, no. 22, p. 2674, 2019.

[8] A. Reigber et al., "First interferometric trials with the airborne digitalbeamforming DBFSAR system," in Proc. 12th Eur. Conf. Synth. Aperture Radar (EUSAR), Jun. 2018, pp. 12-15.

[9] D. Cerutti-Maori, J. Klare, A. R. Brenner, and J. H. G. Ender, "Wide-area traffic monitoring with the SAR/GMTI system PAMIR," IEEE Trans. Geosci. Remote Sens., vol. 46, no. 10, pp. 3019-3030, Oct. 2008.

[10] A. Reigber et al., "Current status of DLR's new F-SAR sensor," in Proc. 8th Eur. Conf. Synth. Aperture Radar (EUSAR), Jun. 2010, pp. $1078-1081$.

[11] A. Reigber et al., "Very-high-resolution airborne synthetic aperture radar imaging: Signal processing and applications," Proc. IEEE, vol. 101, no. 3, pp. 759-783, Mar. 2013

[12] J. H. G. Ender, C. H. Gierull, and D. Cerutti-Maori, "Improved space-based moving target indication via alternate transmission and receiver switching," IEEE Trans. Geosci. Remote Sens., vol. 46, no. 12 , pp. 3960-3974, Dec. 2008.

[13] R. Kohlleppel, "Ground moving target tracking with space-time adaptive radar," Ph.D. dissertation, Dept. Sci. Technol., Univ. Siegen, Siegen, Germany, 2014. Accessed: Jul. 27, 2020. [Online]. Available: http://publica.fraunhofer.de/documents/N-350001.html

[14] W. L. Melvin, "A STAP overview," IEEE Aerosp. Electron. Syst. Mag., vol. 19, no. 1, pp. 19-35, Jan. 2004.
[15] A. Moreira, J. Mittermayer, and R. Scheiber, "Extended chirp scaling algorithm for air- and spaceborne SAR data processing in stripmap and ScanSAR imaging modes," IEEE Trans. Geosci. Remote Sens., vol. 34, no. 5, pp. 1123-1136, Sep. 1996.

[16] T. F. Ayoub, A. M. Haimovich, and M. L. Pugh, "Reduced-rank STAP for high PRF radar," IEEE Trans. Aerosp. Electron. Syst., vol. 35, no. 3, pp. 953-962, Jul. 1999.

[17] G. K. Borsari, "Mitigating effects on STAP processing caused by an inclined array," in Proc. IEEE Radar Conf., Challenges Radar Syst. Solutions, May 1998, pp. 135-140.

[18] C. Wu, J. C. Curlander, and A. Di Cenzo, "Determination of spacecraft attitude using synthetic aperture radar data," Amer. Inst. Aeronaut. Astronaut., vol. 31, no. 7, pp. 57-60, 1980.

[19] R. Bamler, "Doppler frequency estimation and the Cramér-Rao bound," IEEE Trans. Geosci. Remote Sens., vol. 29, no. 3, pp. 385-390, May 1991.

[20] J. A. Nelder and R. Mead, "A simplex method for function minimization," Comput. J., vol. 7, no. 4, pp. 308-313, Jan. 1965.

[21] M. J. D. Powell, "An efficient method for finding the minimum of a function of several variables without calculating derivatives," Comput. J., vol. 7, no. 2, pp. 155-162, Feb. 1964.

[22] C. H. Gierull, "Moving target detection with along-track SAR interferometry," Defence R\&D Canada, Ottawa, ON, Canada, Tech. Rep. DRDC Ottawa TR 2002-084, Aug. 2002.

[23] D. Cerutti-Maori, C. H. Gierull, and J. H. G. Ender, "Experimental verification of SAR-GMTI improvement through antenna switching," IEEE Trans. Geosci. Remote Sens., vol. 48, no. 4, pp. 2066-2075, Apr. 2010.

[24] S. V. Baumgartner and G. Krieger, "Fast GMTI algorithm for traffic monitoring based on a priori knowledge," IEEE Trans. Geosci. Remote Sens., vol. 50, no. 11, pp. 4626-4641, Nov. 2012.

[25] A. B. C. Silva and S. V. Baumgartner, "Novel post-Doppler STAP with a priori knowledge information for traffic monitoring applications: Basic idea and first results," Adv. Radio Sci., vol. 15, pp. 77-82, Apr. 2017.

[26] S. V. Baumgartner, "TRAMRAD GMTI-experimente mit F-SAR," German Aerosp. Center, Oberpfaffenhofen, Germany, Tech. Rep. TRAMRAD-DLR-PD-411, Oct. 2006.

[27] L. Yang, T. Wang, and Z. Bao, "Ground moving target indication using an InSAR system with a hybrid baseline," IEEE Geosci. Remote Sens. Lett., vol. 5, no. 3, pp. 373-377, Jul. 2008.

[28] A. B. C. Silva, S. V. Baumgartner, and A. Moreira, "Fast post-Doppler STAP with road map for traffic monitoring," Adv. Radio Sci., vol. 17, pp. 137-143, Sep. 2019.

[29] C. H. Gierull, "Statistical analysis of multilook SAR interferograms for CFAR detection of ground moving targets," IEEE Trans. Geosci. Remote Sens., vol. 42, no. 4, pp. 691-701, Apr. 2004.

[30] C. H. Gierull, I. Sikaneta, and D. Cerutti-Maori, "Two-step detector for RADARSAT-2's experimental GMTI mode," IEEE Trans. Geosci. Remote Sens., vol. 51, no. 1, pp. 436-454, Jan. 2013.

[31] (2018). OpenStreetMap. Accessed: Nov. 10, 2018. [Online]. Available: http://www.openstreetmap.org

[32] T. Farr et al., "The shuttle radar topography mission," Rev. Geophys., vol. 45, no. 2, pp. 1-33, Jun. 2007.

[33] A. B. C. da Silva and S. V. Baumgartner, "A priori knowledge-based STAP for traffic monitoring applications: First results," in Proc. 11th Eur. Conf. Synth. Aperture Radar (EUSAR), Jun. 2016, pp. 211-215.

[34] R. Klemm, Principles of Space-Time Adaptive Processing, 3rd ed. London, U.K.: The Institution of Engineering and Technology (IET), 2006.

[35] J. R. Guerci, Space-Time Adaptive Processing for Radar, no. 1, 1st ed. Norwood, MA, USA: Artech House, 2003.

[36] R. C. DiPietro, "Extended factored space-time processing for airborne radar systems," in Proc. Conf. Rec. 26th Asilomar Conf. Signals, Syst. Comput., Oct. 1992, pp. 425-430.

[37] F. C. Robey, D. R. Fuhrmann, E. J. Kelly, and R. Nitzberg, "A CFAR adaptive matched filter detector," IEEE Trans. Aerosp. Electron. Syst., vol. 28, no. 1, pp. 208-216, Jan. 1992.

[38] A. B. C. da Silva, S. V. Baumgartner, and G. Krieger, "Training data selection and update strategies for airborne post-Doppler STAP,' IEEE Trans. Geosci. Remote Sens., vol. 57, no. 8, pp. 5626-5641, Aug. 2019.

[39] I. S. Reed, J. D. Mallett, and L. E. Brennan, "Rapid convergence rate in adaptive arrays," IEEE Trans. Aerosp. Electron. Syst., vol. AES-10, no. 6, pp. 853-863, Nov. 1974.

[40] F. Gini and M. Greco, "Covariance matrix estimation for CFAR detection in correlated heavy tailed clutter," Signal Process., vol. 82, no. 12, pp. 1847-1859, Dec. 2002. 


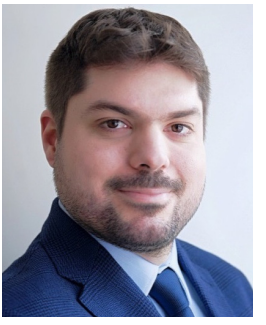

André Barros Cardoso da Silva was born in Taubaté, Brazil, in 1986. He received the B.S. degree (summa cum laude) in telecommunication engineering from the University of Taubaté, Taubaté, in 2009, the M.S. degree in electronic engineering from the Aeronautics Technological Institute, São José dos Campos, Brazil, in 2013, and the Dr.Ing. (Ph.D.) degree (magna cum laude) in electrical engineering from the Karlsruhe Institute of Technology, Karlsruhe, Germany, in 2019.

From 2005 to 2009, he joined the Brazilian National Institute for Space Research (INPE), São José dos Campos, as an Engineering Intern, where he was involved in data collection platforms for the Brazilian Environmental Data Collection System satellites. From 2010 to 2014, he was with the Brazilian Antarctic Program (PROANTAR) as a Research Engineer on five missions during the summer seasons (228 days in total), where he focused on the activation and maintenance of atmospheric radars and data collection systems for environmental monitoring in the Brazilian Antarctic Station "Comandante Ferraz" (EACF), Antarctica. Since 2014, he has been with Microwaves and Radar Institute, German Aerospace Center (DLR-HR), Weßling, Germany, first as Ph.D. Fellow and then as a Research Scientist. His research interests include moving target indication over land and ocean using synthetic aperture radars, along-track interferometry, and space-time adaptive processing methodologies.

Dr. da Silva received the honorable mention in telecommunication and the CREA-SP Professional Formation Award granted by the Brazilian Regional Council of Engineering and Architecture of São Paulo state for first-class students in engineering courses in 2010.

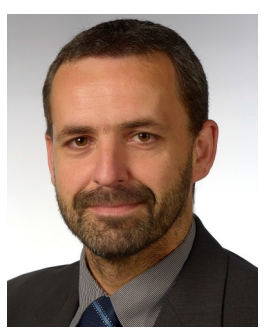

Stefan Valentin Baumgartner (Member, IEEE) received the Dipl.Ing. (M.S.) degree (Hons.) in electrical and communication engineering from the Graz University of Technology, Graz, Austria, in 2004, and the Dr.Ing. (Ph.D.) degree (Hons.) in electrical and communication engineering from the Karlsruhe Institute of Technology (KIT), Karlsruhe, Germany, in 2014.

Since 2004, he has been with Microwaves and Radar Institute, German Aerospace Center (DLR), Weßling, Germany. As a Scientist and Project Manager, he was and is involved in several national and international projects related to moving target indication (MTI) with single-channel and multichannel airborne and spaceborne synthetic aperture radar (SAR) sensors. $\mathrm{He}$ also planned the first successful bistatic experiment between DLR's airborne radar sensor F-SAR and the German radar satellite TerraSAR$\mathrm{X}$, whose results were presented at the European Conference on Synthetic Aperture Radar (EUSAR) in 2008. From 2012 to 2017, he had the technical lead of the successful European Space Agency project "Study of MultiChannel Ka-Band SARs for Ground Moving Target Indication." Since 2010, he has been lecturing on "SAR Moving Target Techniques" for the CarlCranz-Gesellschaft (CCG). Since 2016, he has also been active at FriedrichAlexander-University Erlangen-Nuremberg, Erlangen, Germany. He has the lead of a maritime security project, with a focus on the development of novel ship detection and inverse SAR (ISAR) algorithms using airborne radars operated along the arbitrary flight tracks. He successfully participated in the DLR Graduate Program, a DLR qualification initiative that accompanies the doctoral phase. From 2016 to 2018, he attended and successfully completed the DLR Talent Management Program, a high-quality and very individual personnel development tool that provides outstanding employees with intensive support over the course of two years. He has authored or coauthored more than 15 peer-reviewed journal articles, about 60 conference papers, and an invited book chapter on multichannel SAR for ground moving target indication for the Academic Press Library in Signal Processing. He holds one patent in the field of SAR-MTI. His research interests include the development of novel MTI algorithms for road and maritime traffic monitoring applications using single-channel and multichannel airborne and spaceborne SARs, along-track interferometry, space-time adaptive processing (STAP), ISAR imaging, digital beamforming on receive, and time-frequency analysis.

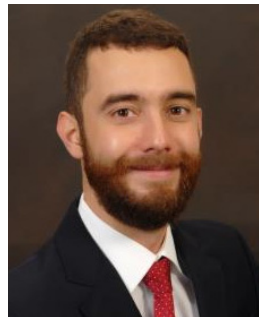

Felipe Queiroz de Almeida received the B.S.E.E. (Hons.) and M.S.E.E. degrees in electronic engineering from the Aeronautics Technological Institute (ITA), São José dos Campos, Brazil, in 2009 and 2011, respectively, and the Dr.Ing. (Ph.D.) degree (Hons.) in electrical engineering from the Karlsruhe Institute of Technology (KIT), Karlsruhe, Germany, in 2018. He developed his bachelor's thesis at Microwaves and Radar Institute, German Aerospace Center (DLR-HR), Weßling, Germany.

In 2009, he was a Research Intern with the Microwaves and Radar Institute, DLR-HR. From 2011 to 2014, he was with Bradar Indústria S.A., São José dos Campos, where he worked on software development for synthetic aperture radar applications. Since November 2014, he has been with DLR-HR, first as a Ph.D. Student and then as a Researcher. His research interests include radar signal processing and digital beamforming, in particular beamforming techniques for high-resolution wide-swath synthetic aperture radar (SAR).

Dr. Queiroz de Almeida received the EADS Argus Student Award 2010 for his bachelor's thesis, the Franz-Xaver-Erlacher Award for Young Scientists in 2015, and the ITG Award in 2019 for the best research paper.

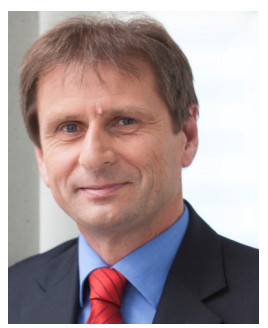

Gerhard Krieger (Fellow, IEEE) received the Dipl.Ing. (M.S.) and Dr.Ing. (Ph.D.) (Hons.) degrees in electrical and communication engineering from the Technical University of Munich, Munich, Germany, in 1992 and 1999, respectively.

From 1992 to 1999, he was with Ludwig Maximilians University, Munich, where he conducted multidisciplinary research on neuronal modeling and nonlinear information processing in biological and technical vision systems. Since 1999, he has been with the Microwaves and Radar Institute, German Aerospace Center (DLR), Weßling, Germany, where he started as a Research Associate developing signal processing algorithms for a novel forward-looking radar system using digital beamforming on receive. From 2001 to 2007, he led the New SAR Missions Group that pioneered the development of advanced bistatic and multistatic radar systems, such as TanDEM-X, as well as innovative multichannel synthetic aperture radar (SAR) techniques and algorithms for high-resolution wide-swath SAR imaging. Since 2008, he has been the Head of the Radar Concepts Department, which hosts about 40 scientists focusing on new SAR techniques, missions, and applications. He has also been serving as a Mission Engineer for TanDEM-X and made major contributions to the development of the Tandem-L mission concept, where he led the Phase-0 and Phase-A studies. Since 2019, he has been a Professor with the Friedrich-Alexander-University Erlangen, Erlangen, Germany. He has authored or coauthored more than 90 peer-reviewed journal articles, 9 invited book chapters, about 400 conference papers, and more than 15 patents.

Dr. Krieger has been an Associate Editor of the IEEE TRANS ACTIONS ON Geoscience ANd REMOTE SENSING since 2012. In 2014, he served as the Technical Program Chair for the European Conference on Synthetic Aperture Radar and a Guest Editor for the IEEE JOURNAL OF SELECTED TOPICS IN ApPlied EARTh ObSERVATIONS AND REMOTE SEnSing. He has received several national and international awards, including the W.R.G. Baker Prize Paper Award from the IEEE Board of Directors, two transactions prize paper awards of the IEEE Geoscience and Remote Sensing Society, and two best paper awards at the European Conference on Synthetic Aperture Radar. 